\title{
Hybrid ARQ in Multiple-Antenna Slow Fading Channels: Performance Limits and Optimal Linear Dispersion Code Design
}

\author{
Cong Shen Michael P. Fitz ${ }^{* \dagger}$
}

November 15, 2018

\begin{abstract}
This paper focuses on studying the fundamental performance limits and linear dispersion code design for the MIMO-ARQ slow fading channel. Optimal average rate of well-known HARQ protocols is analyzed. The optimal design of space-time coding for the MIMO-ARQ channel is discussed. Information-theoretic measures are used to optimize the rate assignment and derive the optimum design criterion, which is then used to evaluate the optimality of existing space-time codes. A different design criterion, which is obtained from the error probability analysis of space-time coded MIMO-HARQ, is presented. Examples are studied to reveal the gain of ARQ feedback in space-time coded MIMO systems.
\end{abstract}

Index Terms: Space-time code, Hybrid ARQ, Multiple-antenna channels, Linear Dispersion Code.

\section{Introduction}

Hybrid ARQ (HARQ) techniques combine the automatic-repeat-request (ARQ) feedback with the forward error correction (FEC) codes to achieve better reliability and higher

${ }^{*}$ The material in this paper was presented in part at the 42nd Annual Asilomar Conference on Signals, Systems, and Computers, Pacific Grove, CA, USA, Oct. 2008.

${ }^{\dagger}$ Cong Shen is with the Department of Electrical Engineering, University of California Los Angeles (UCLA), Los Angeles, CA 90095, USA. Email: congshen@ee.ucla.edu. Michael P. Fitz was with the Department of Electrical Engineering, University of California Los Angeles (UCLA), Los Angeles, CA 90095, USA. He is now with Northrop Grumman Space Technology, Redondo Beach, CA 90278, USA. Email: Michael.Fitz@ngc. com. 
throughput [1]. They are widely used in most of the contemporary communication systems, as the delay tolerance of many data services allows using retransmission to recover erroneous packets. A good summary of the progress of HARQ schemes is presented in [2]. From an information-theoretic perspective, HARQ systems can be viewed as channel with sequential feedback, and there has been some research on the fundamental limits of this channel. An information-theoretic throughput and delay analysis of several HARQ schemes in a Gaussian collision channel is presented in [3]. Throughput analysis of incremental redundancy HARQ in the block-fading additive white Gaussian noise (AWGN) channel is carried out in [4]. Optimal average rate performance of a scalar slow fading channel with ARQ feedback is done in $[5,6]$.

Most of the aforementioned research is restricted to scalar channels. A natural question is how to incorporate the design of HARQ into multiple-input-multiple-output (MIMO) systems, and it has sparked many research activities. Most existing literature regarding MIMO-HARQ design falls into the following two categories. One is the information-theoretic analysis, for which the focus is on extending the Diversity-Multiplexing Tradeoff (DMT) framework [7] to consider ARQ feedback [8-10]. The other is the joint design of MIMO transmission and ARQ feedback. Receiver processing of ARQ retransmissions is studied in $[11,12]$. It has been shown that multiple ARQ packets combining is non-trivial in a MIMO setting, especially when soft-output is desired [13]. For the transmitter design, two different approaches have been taken to exploit the additional spatial degrees of freedom. One is to study the bits/symbols rearrangement for retransmissions to exploit diversity [14-17], and the other is to explore linear precoder design $[18,19]$.

Despite the many efforts in studying MIMO-HARQ design, there are still some important problems left unanswered. From the perspective of information-theoretic analysis, the DMTbased approach focuses only on the high Signal-to-Noise Ratio (SNR) asymptotics and gives the tradeoff between multiplexing gain (pre-log), diversity gain (error exponent) and ARQ delay. It also requires a family of space-time codes (STC) whose rates scale with the SNR. In practice, however, one is also interested in designing a fixed-rate STC that operates well within a range of finite SNRs. It is unclear what is the optimal MIMO-HARQ throughput in this scenario, and how to design the optimal STC to achieve it. For the practical HARQ retransmission protocol design, the aforementioned works provide separate and ad hoc designs. There is no general framework that unifies the existing results and guides the optimal design.

This paper is devoted to solving these problems. We begin by introducing the system model in Sec. 2, The performance limits of existing HARQ protocols in a MIMO setting are investigated in Sec. 3. Sec. 4 studies the fundamental average rate limit of STC-based MIMO-HARQ protocols, and gives the optimal STC design criterion. Many existing STCs are re-visited under this framework, both analytically and numerically. A different approach based on the error probability analysis is given in Sec. 5, where the so-called $n$-th pairwise error probability plays a key role in the analysis. Optimal STC design criterion based on the error probability analysis is proposed and exemplary STCs are studied. Finally, Sec. 6 concludes the paper. 
Throughout the paper the following notations will be used. Matrices and vectors are denoted with bold capital and lowercase letters, respectively. $\mathbf{A}(i, j)$ is the $(i, j)$-th element of the matrix $\mathbf{A}$, and $\mathbf{a}(i)$ is the $i$-th element of the vector $\mathbf{a}$. $\mathbb{E}[\cdot]$ denotes the expectation of a random variable. $a^{*}$ denotes the conjugate of the complex number $a$, $\operatorname{Re}\{a\}$ is the real part of $a$, and $\mathbf{a}^{t}$ is the transpose of vector $\mathbf{a}$. We use $\operatorname{Tr}(\mathbf{A})$ and $\|\mathbf{A}\|_{F}$ to denote the trace and Frobenius norm of matrix $\mathbf{A}$, respectively. $\mathbf{A}^{H}$ and conj $(\mathbf{A})$ are the Hermitian and the conjugate of the complex matrix A, respectively. The Kronecker product operation of two matrices is denoted by $\otimes$, and $\operatorname{vec}(\cdot)$ is the vectorization operation. Finally, $|\mathcal{W}|$ denotes the cardinality of the set $\mathcal{W}$.

\section{System model}

In this section, the MIMO channel model with ARQ feedback is described, and existing HARQ protocols that will be analyzed in Sec. 3 are introduced.

\subsection{MIMO channel with ARQ feedback}

This paper studies a single-user multiple-antenna wireless system equipped with $L_{t}$ transmit and $L_{r}$ receive antennas. It is assumed that there is an error-free and delay-free ARQ feedback link, which indicates to the transmitter the successful decoding by acknowledgement (ACK) and failed decoding by negative acknowledgement (NACK). The maximum number of ARQ transmissions is denoted by $N$, which is also called the maximum allowable ARQ rounds in the literature. With this constraint, if after a total of $N$ transmissions the receiver still cannot decode the message successfully, no further attempt will be tried and a decoding failure is declared.

It is assumed there is a very large pool of information messages available at the transmitter. As soon as the transmission of current message (including possible retransmissions) is done, the next message is encoded and transmitted immediately. Consider a set of uniformly distributed messages $\mathcal{W}$. For the transmission of each message, the information message $w \in \mathcal{W}$ is sent to a MIMO-HARQ encoder, which generates $N$ matrix sub-codewords $\left\{\mathbf{X}_{n}(w)\right\}_{n=1}^{N}$, each corresponds to one ARQ round. Sub-codeword $\mathbf{X}_{n}(w)$ will be transmitted in the $n$-th ARQ round if the decoding fails at the $(n-1)$-th round. Denote $\mathbf{X}^{(n)}(w) \doteq\left[\mathbf{X}_{1}(w), \cdots, \mathbf{X}_{n}(w)\right], \forall n=1, \cdots, N$, which is the overall codeword after the $n$-th ARQ round. The receiver performs optimum decoding based on all the received packets in ARQ round $1, \cdots, n$. Each sub-codeword satisfies $\mathbf{X}_{n}(w) \in \mathcal{C}^{L_{t} \times L_{n} / r_{n}}$, where $L_{n} / r_{n}$ denotes the length of the sub-codeword, $r_{n}$ is determined by the "coding rate" of the space-time structure as $r_{n} \doteq \frac{K_{n}}{T_{n}}$ where $K_{n}$ and $T_{n}$ are the number of symbols and the time slots per space-time codeword, respectively, and $L_{n}$ is the capacity-achieving channel code length. 
With this model, the overall transmission rate in the $n$-th ARQ round is

$$
R^{(n)}=\frac{\log _{2}|\mathcal{W}|}{\sum_{i=1}^{n} L_{i} / r_{i}}, \forall n=1, \cdots, N .
$$

The MIMO channel $\mathbf{H} \in \mathcal{C}^{L_{r} \times L_{t}}$ is assumed to be a random matrix with independent and identically distributed (i.i.d.) complex circularly symmetric Gaussian entries with unit variance. The channel matrix $\mathbf{H}$ is assumed to be constant within the channel coherence time ( $T_{c}$ symbols), and changes independently to a different value in the next coherence block. Depending on the relationship between $T_{c}$ and $\left\{L_{n} / r_{n}\right\}_{n=1}^{N}$, different MIMO-HARQ models have been studied in the literature $[8,10]$. In this work, a slow fading MIMO channel model is considered, where $T_{c} \gg \sum_{n=1}^{N} L_{n} / r_{n}$. This means the MIMO channel remains unchanged throughout all possible retransmissions. This models the worst-case scenario compared to $[8,10]$, as there is no time diversity to exploit by retransmissions.

With the described model, the received signal corresponding to the $n$-th transmission can be written as

$$
\mathbf{Y}_{n}=\sqrt{\frac{\mathrm{SNR}}{L_{t}}} \mathbf{H X}_{n}+\mathbf{Z}_{n}
$$

and the overall received signal after the $n$-th ARQ round is

$$
\mathbf{Y}^{(n)}=\sqrt{\frac{\mathrm{SNR}}{L_{t}}} \mathbf{H} \mathbf{X}^{(n)}+\mathbf{Z}^{(n)},
$$

where $\mathbf{Y}^{(n)} \doteq\left[\mathbf{Y}_{1}, \cdots, \mathbf{Y}_{n}\right]$ and $\mathbf{X}^{(n)}, \mathbf{Z}^{(n)}$ are similarly defined; the additive noise $\mathbf{Z}^{(n)}$ has i.i.d. entries $\mathbf{Z}^{(n)}(i, j) \sim \mathcal{C} \mathcal{N}(0,1)$. The overall transmit codewords $\mathbf{X}^{(N)}(w)$ are normalized to satisfy the average power constraint 1

$$
\frac{1}{\sum_{n=1}^{N} L_{n} / r_{n}} \mathbb{E}\left\|\mathbf{X}^{(N)}(w)\right\|_{F}^{2} \leq L_{t}
$$

where the expectation is over all possible codewords. Therefore, the average SNR per receive antenna is SNR. The receiver is assumed to have perfect knowledge of $\mathbf{H}$. This is a reasonable assumption since the slowly varying nature of the channel helps receiver channel estimation. The transmitter is assumed to have no knowledge of $\mathbf{H}$ prior to the transmission, but it should be noted that ARQ retransmissions essentially inform partial CSI to the transmitter in a sequential fashion [6]. Fig. 1 illustrates the system model under consideration.

\subsection{Hybrid ARQ strategies}

HARQ incorporates FEC coding into ARQ feedback to enhance the retransmission performance. Depending on transmitter's retransmission strategy and receiver's decoding from

\footnotetext{
${ }^{1}$ The transmit power constraint will be discussed in more detail when the optimal LDC design for MIMOHARQ is presented in Sec. 4.
} 


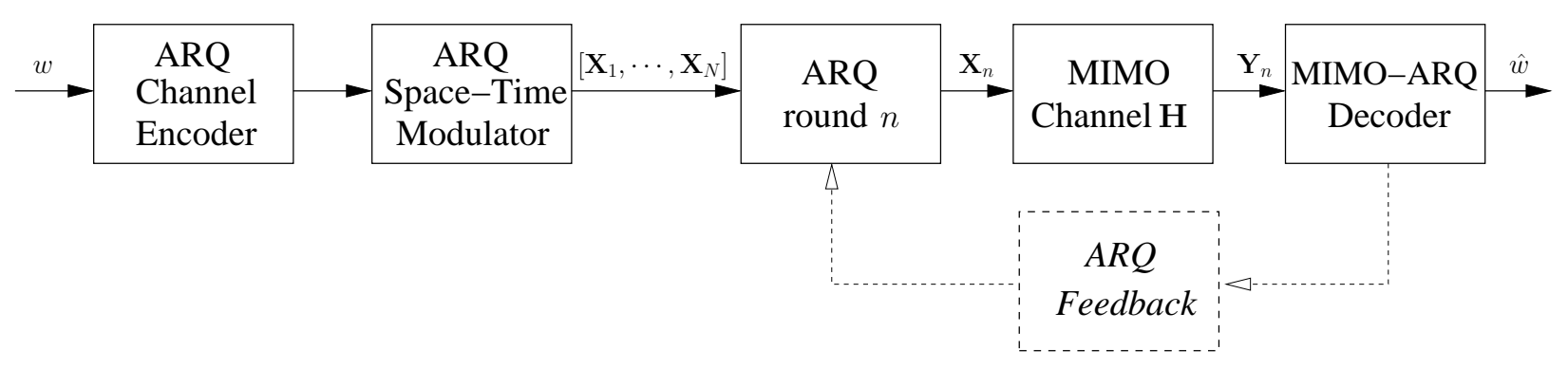

Figure 1: The MIMO-HARQ system model.

multiple packets, several efficient protocols have been proposed and studied. In this work, two traditional stop-and-wait HARQ protocols are considered:

1) Incremental Redundancy (IR). This is a code combining scheme [20]. At the transmitter, information message is encoded into an overall codeword of length $\sum_{n=1}^{N} L_{n}$, and then the codeword is serially punctured into $N$ sub-codewords each with length $L_{n}$. These sub-codewords are mapped into the transmit matrix codewords $\left[\mathbf{X}_{1}, \cdots, \mathbf{X}_{N}\right]$. At the $n$-th transmission, $\mathbf{X}_{n}$ is sent through the channel as additional (when $n>1$ ) redundancy symbols to reduce the transmission rate. The receiver tries to decode the message based on all the packets it receives up until round $n$.

2) Chase Combining (CC). This belongs to the category of diversity combining [20]. Upon each retransmission request, the transmitter simply repeats the same packet: $\mathbf{X}_{n}=\mathbf{X}_{1}, \forall n=2, \cdots, N$. Different diversity combining schemes can be used at the receiver, among which the Chase combining [21], which essentially is a maximum ratio combining of all the received packets in the scalar channel, gives the best performance.

These well-known HARQ protocols will be analyzed in a MIMO setting and the optimal performance is reported in Section 3 .

Traditional HARQ protocols are designed to extract time diversity in a scalar fading channel. The performance depends critically on the channel variation over retransmissions. The slow fading channel model considered in this work allows no time diversity for the HARQ protocols to exploit, and hence packet-combining-based schemes have limited performance [6]. Multiple antenna system provides additional dimensions for spatial diversity, which is not effectively exploited by traditional protocols. On the other hand, although provides the best performance, IR has higher complexity due to the generation of rate-compatible channel codes or the rateless codes. Both problems call for the design of HARQ protocols that jointly consider the space-time structure to exploit spatial diversity and low-complexity ARQ retransmissions. This is the focus of Section 4 and 5 .

The figure of merit in this paper is the long-term throughput, and an information-theoretic view is taken for the MIMO-HARQ design problem. The throughput of a HARQ protocol is defined [20] as the average number of bits accepted by the receiver in the time it takes to send 
the data packet. As discussed in [6], how to compute the throughput depends on the specific applications and the assumptions on how to use the channel. Typical derivation involves the evaluation of average transmission time. Renewal theory has been successfully applied to this problem $[3,22]$. However, this formulation leads to the problem that the empirical channel distribution does not match the true channel statistics, and the reason is that the length of channel uses is determined by the instantaneous channel state. This problem has been discussed in [6,23], and it has been shown [6] that for some applications, average rate [24-26] gives the long-term successful communication rate and is a more reasonable metric. The average rate is chosen as the performance measure in this work.

\section{Performance limits of existing HARQ protocols}

This section studies the optimal average rate performance where the existing HARQ protocols are directly applied in a vector channel. We will first derive the average rate for general HARQ systems, and then apply the result to IR and CC. The general average rate expression is a fundamental result and will also be useful in developing optimal space-time HARQ in Section 4 .

Before analyzing the average rate, some important events are defined and the associated probabilities are studied. Due to the randomness of the channel matrix $\mathbf{H}$ and the limit $N$ on the ARQ deadline, the successful communication rate $R$ is a random variable. Define two events for ARQ round $n, \forall n=1, \cdots, N$ :

$$
\begin{aligned}
& \mathcal{A}_{n}=\text { Decoding at the end of ARQ round } n \text { is successful; } \\
& \mathcal{S}_{n}=\text { ARQ round } n \text { is activated. }
\end{aligned}
$$

There are two important observations about these events, both originated from an information theoretic viewpoint2. First, a decoding failure is equivalent to a channel outage, i.e., the instantaneous channel capacity cannot support the current transmission rate. This is because long-blocklength capacity-achieving channel coding "averages out" the additive noise. Thus one has

$$
\operatorname{Pr}\left\{\mathcal{A}_{n}\right\}=\operatorname{Pr}\left\{C^{(n)}\left(\mathbf{H}_{\text {eq }}^{(n)}\right) \geq R^{(n)}\right\} .
$$

where $C^{(n)}\left(\mathbf{H}_{\mathrm{eq}}^{(n)}\right)$ is the equivalent channel capacity at ARQ round $n ; R^{(n)}$ is the overall communication rate at round $n$, which is given in Equation (11). The second observation is that additional transmissions can only increase the successful decoding probability, via either increasing $C^{(n)}\left(\mathbf{H}_{\mathrm{eq}}^{(n)}\right)$ or reducing $R^{(n)}$, or both. This observation suggests

$$
\mathcal{A}_{n-1} \subseteq \mathcal{A}_{n}
$$

and hence

$$
\begin{aligned}
\operatorname{Pr}\left\{\mathcal{S}_{n}\right\} & =\operatorname{Pr}\left\{\overline{\mathcal{A}_{1}}, \cdots, \overline{\mathcal{A}_{n-1}}\right\} \\
& =\operatorname{Pr}\left\{\overline{\mathcal{A}_{n-1}}\right\} .
\end{aligned}
$$

\footnotetext{
${ }^{2}$ As one will see in Sec. 5, finite coding length leads to some different conclusions for the HARQ design.
} 
These observations play an important role in analyzing the information-theoretic performance limits of MIMO-HARQ.

With the definitions (5) and (6), the successful communication rate $R$ can be expressed as

$$
\begin{aligned}
& R= \begin{cases}R^{(n)}, & \text { if }\left\{\mathcal{S}_{n}, \mathcal{A}_{n}\right\}, \forall n=1, \cdots, N \\
0, & \text { if } \mathcal{A}_{N}\end{cases} \\
& = \begin{cases}R^{(n)}, & \text { if }\left\{\overline{\mathcal{A}_{n-1}}, \mathcal{A}_{n}\right\}, \forall n=1, \cdots, N \\
0, & \text { if } \overline{\mathcal{A}_{N}}\end{cases}
\end{aligned}
$$

where the second equality comes from (9), and the convention $\operatorname{Pr}\left\{\mathcal{A}_{0}\right\}=0$ is used. The average rate is then

$$
\begin{aligned}
\bar{R} & =\sum_{n=1}^{N} R^{(n)} \operatorname{Pr}\left\{\overline{\mathcal{A}_{n-1}}, \mathcal{A}_{n}\right\} \\
& =\sum_{n=1}^{N} R^{(n)} \operatorname{Pr}\left\{C^{(n-1)}\left(\mathbf{H}_{\mathrm{eq}}^{(n-1)}\right)<R^{(n-1)}, C^{(n)}\left(\mathbf{H}_{\mathrm{eq}}^{(n)}\right) \geq R^{(n)}\right\},
\end{aligned}
$$

where we define $R^{(0)}=\infty$ (since the denominator in $(\mathbb{1})$ is zero) and $C^{(0)}\left(\mathbf{H}_{\text {eq }}\right)=0$. A different expression can be obtained if one notices that

$$
\operatorname{Pr}\left\{\overline{\mathcal{A}_{n-1}}, \mathcal{A}_{n}\right\}=\operatorname{Pr}\left\{\mathcal{A}_{n}\right\}-\operatorname{Pr}\left\{\mathcal{A}_{n-1}\right\},
$$

which is true since $\mathcal{A}_{n-1} \subseteq \mathcal{A}_{n}$. The average rate expression (12) then can be written as

$$
\begin{aligned}
\bar{R} & =\sum_{n=1}^{N} R^{(n)}\left(\operatorname{Pr}\left\{\mathcal{A}_{n}\right\}-\operatorname{Pr}\left\{\mathcal{A}_{n-1}\right\}\right) \\
& =\sum_{n=1}^{N}\left(R^{(n)}-R^{(n+1)}\right) \operatorname{Pr}\left\{\mathcal{A}_{n}\right\} \\
& =\sum_{n=1}^{N}\left(R^{(n)}-R^{(n+1)}\right) \operatorname{Pr}\left\{C^{(n)}\left(\mathbf{H}_{\text {eq }}^{(n)}\right) \geq R^{(n)}\right\},
\end{aligned}
$$

where we define $R^{(N+1)}=0$ (since the retransmission stops after the $n$-th round). It should be noted that the term $R^{(n)}-R^{(n+1)}$ denotes the rate decrease in the $(n+1)$-th transmission.

The equivalent channel capacity $C^{(n)}\left(\mathbf{H}_{\mathrm{eq}}^{(n)}\right)$ at ARQ round $n$ is determined not only by the instantaneous channel matrix $\mathbf{H}$, but also by the specific HARQ protocol and the transmit covariance matrices $\left\{\mathbf{K}_{i}\right\}_{i=1}^{n}$, where $\mathbf{K}_{i}$ is the transmit covariance matrix at ARQ round $i$ and could be different over retransmissions. As one shall see in the sequel, these degrees of freedom shall be carefully exploited in the MIMO-HARQ protocol design.

With the general average rate expressions (13) and (16), we proceed to study IR and CC, respectively. 


\subsection{Incremental Redundancy}

The average rate for general IR can be obtained directly from (16):

$$
\bar{R}_{\mathrm{ir}}=\sum_{n=1}^{N}\left(R^{(n)}-R^{(n+1)}\right) \operatorname{Pr}\left\{C_{\mathrm{ir}}^{(n)}\left(\mathbf{H}_{\mathrm{eq}, \mathrm{ir}}^{(n)}\right) \geq R^{(n)}\right\},
$$

and the remaining problem is to analyze the capacity of IR after each ARQ round. The capacity for a given MIMO channel $\mathbf{H}$ and total transmit covariance matrix $\mathbf{K}$ is known to be [27]

$$
C_{\text {mimo }}(\mathbf{H}, \mathbf{K})=\log \operatorname{det}\left(\mathbf{I}_{L_{r}}+\mathbf{H} \mathbf{K} \mathbf{H}^{H}\right)
$$

where $\mathbf{K}$ has to satisfy the power constraint 3

$$
\operatorname{Tr}(\mathbf{K}) \leq \text { SNR. }
$$

Assuming transmit covariance matrix $\mathbf{K}_{n}$ is used in ARQ round $n$, the overall IR capacity where different portions have different covariance matrices is a TDMA-type one:

$$
\begin{aligned}
C_{\mathrm{ir}}^{(n)}\left(\mathbf{H}_{\mathrm{eq}, \mathrm{ir}}^{(n)}\right) & =\frac{\sum_{i=1}^{n} C_{\text {mimo }}\left(\mathbf{H}, \mathbf{K}_{i}\right) L_{i} / r_{i}}{\sum_{i=1}^{n} L_{i} / r_{i}} \\
& =\sum_{i=1}^{n}\left(\frac{R^{(n)}}{R^{(i)}}-\frac{R^{(n)}}{R^{(i-1)}}\right) C_{\text {mimo }}\left(\mathbf{H}, \mathbf{K}_{i}\right) .
\end{aligned}
$$

where (20) is obtained from (11).

The general IR average rate maximization problem can now be formulated as

$$
\begin{array}{ll}
\underset{\left\{\mathbf{K}_{n}, R_{n}\right\}_{n=1}^{N}}{\operatorname{maximize}} & \sum_{n=1}^{N}\left(R^{(n)}-R^{(n+1)}\right) \operatorname{Pr}\left\{\sum_{i=1}^{n}\left(\frac{R^{(n)}}{R^{(i)}}-\frac{R^{(n)}}{R^{(i-1)}}\right) C_{\text {mimo }}\left(\mathbf{H}, \mathbf{K}_{i}\right) \geq R^{(n)}\right\} \\
\text { subject to } & R^{(N)} \geq 0 \\
& R^{(n)} \leq R^{(n-1)}, \forall n=1, \cdots, N \\
& \operatorname{Tr}\left(\mathbf{K}_{n}\right) \leq \mathrm{SNR}, \forall n=1, \cdots, N
\end{array}
$$

Solving this problem is extremely difficult, which comes mainly from the search of optimal covariance matrices $\left\{\mathbf{K}_{n}\right\}_{n=1}^{N}$. Evaluating the objective function in Problem (21) requires the distribution of MIMO capacity $C_{\text {mimo }}(\mathbf{H}, \mathbf{K})$ as a function of the covariance matrix $\mathbf{K}$, which is an unsolved problem. In fact, even the simpler problem of finding the optimal covariance matrix that minimizes the outage probability is still open.

To make progress, this paper will focus on the isotropic Gaussian input distribution over all transmit antennas:

$$
\mathbf{K}_{n}=\mathbf{K}=\frac{\mathrm{SNR}}{L_{t}} \mathbf{I}_{L_{t}} .
$$

\footnotetext{
${ }^{3}$ The constraint (19) assumes that each ARQ round consumes the same average power. One can also dynamically allocate power among ARQ rounds, which leads to a different power constraint and average rate expression. The case for scalar fading channel has been addressed in [6].
} 
This is by no means the optimal solution but very easy to deal with and widely assumed in literature. With (22) the IR capacity is the mutual information of a MIMO channel with isotropic Gaussian input distribution:

$$
\begin{aligned}
C_{\mathrm{ir}}^{(n)}\left(\mathbf{H}_{\mathrm{eq}, \mathrm{ir}}^{(n)}\right) & =C_{\text {mimo }}\left(\mathbf{H}, \frac{\mathrm{SNR}}{L_{t}} \mathbf{I}_{L_{t}}\right) \\
& =\log \operatorname{det}\left(\mathbf{I}_{L_{r}}+\frac{\mathrm{SNR}}{L_{t}} \mathbf{H H}^{H}\right),
\end{aligned}
$$

and the IR average rate maximization problem becomes

$$
\begin{array}{cl}
\underset{\left\{R_{n}\right\}_{n=1}^{N}}{\operatorname{maximize}} & \sum_{n=1}^{N}\left(R^{(n)}-R^{(n+1)}\right) \operatorname{Pr}\left\{C_{\text {mimo }}\left(\mathbf{H}, \frac{\mathrm{SNR}}{L_{t}} \mathbf{I}_{L_{t}}\right) \geq R^{(n)}\right\} \\
\text { subject to } & R^{(N)} \geq 0 \\
& R^{(n)} \leq R^{(n-1)}, \forall n=1, \cdots, N .
\end{array}
$$

For the remaining of this paper, the isotropic Gaussian input distribution is assumed unless the covariance matrix is explicitly specified.

\subsection{Chase Combining}

In CC, each retransmission effectively increases the number of receive antennas by $L_{r}$ via the repetition of previous packet. Thus after ARQ round $n$, the equivalent system is a $\left(L_{t}, n L_{r}\right)$ MIMO with an equivalent channel matrix

$$
\mathbf{H}_{\mathrm{eq}, \mathrm{cc}}^{(n)}=\mathbb{1}_{n} \otimes \mathbf{H}
$$

and

$$
C_{\mathrm{cc}}^{(n)}\left(\mathbf{H}_{\mathrm{eq}, \mathrm{cc}}^{(n)}\right)=\frac{1}{n} C_{\text {mimo }}\left(\mathbf{H}_{\mathrm{eq}, \mathrm{cc}}^{(n)}, \mathbf{K}\right) .
$$

Meanwhile, since each retransmission is a simple packet repetition, the effective rate after ARQ round $n$ is

$$
R^{(n)}=\frac{R}{n}
$$

where $R$ is the rate in ARQ round 1. Hence, the general CC average rate maximization problem is formulated as

$$
\begin{array}{ll}
\underset{\mathbf{K}, R}{\operatorname{maximize}} & \sum_{n=1}^{N} \frac{R}{n} \operatorname{Pr}\left\{C_{\text {mimo }}\left(\mathbf{H}_{\text {eq, cc }}^{(n-1)}, \mathbf{K}\right)<R, C_{\text {mimo }}\left(\mathbf{H}_{\text {eq, cc }}^{(n)}, \mathbf{K}\right) \geq R\right\} \\
\text { subject to } & R \geq 0 \\
& \operatorname{Tr}(\mathbf{K}) \leq \text { SNR. }
\end{array}
$$

Note that due to the simple packet repetition in retransmissions, only one covariance matrix $\mathbf{K}$ and one rate parameter $R$ need to be optimized. If the isotropic Gaussian input distribution is assumed, the problem becomes ( $R \geq 0$ can be dropped):

$$
\underset{R}{\operatorname{maximize}} \sum_{n=1}^{N} \frac{R}{n} \operatorname{Pr}\left\{C_{\text {mimo }}\left(\mathbf{H}_{\text {eq, cc }}^{(n-1)}, \frac{\mathrm{SNR}}{L_{t}} \mathbf{I}_{L_{t}}\right)<R, C_{\text {mimo }}\left(\mathbf{H}_{\text {eq, cc }}^{(n)}, \frac{\mathrm{SNR}}{L_{t}} \mathbf{I}_{L_{t}}\right) \geq R\right\} .
$$


Further simplification is possible if one notices that

$$
\begin{aligned}
\left(\mathbf{H}_{\text {eq }, c c}^{(n)}\right)\left(\mathbf{H}_{\text {eq }, c c}^{(n)}\right)^{H} & =\left(\mathbb{1}_{n} \otimes \mathbf{H}\right)\left(\mathbb{1}_{n}^{T} \otimes \mathbf{H}^{H}\right) \\
& =\mathbb{1}_{n} \mathbb{1}_{n}^{T} \otimes \mathbf{H} \mathbf{H}^{H},
\end{aligned}
$$

and

$$
C_{\text {mimo }}\left(\mathbf{H}_{\text {eq, cc }}^{(n)}, \frac{\mathrm{SNR}}{L_{t}} \mathbf{I}_{L_{t}}\right)=\log \operatorname{det}\left(\mathbf{I}_{n L_{r}}+\frac{\mathrm{SNR}}{L_{t}} \mathbb{1}_{n} \mathbb{1}_{n}^{T} \otimes \mathbf{H H}^{H}\right) .
$$

\subsection{Examples}

To evaluate the performance of IR and CC, several exemplary MIMO configurations are considered and the corresponding optimal average rate performance (24) (29) is compared in this section.

We start with the case of $L_{r}=1$ (MISO) 4 , which is easy to analyze as the received signal expands only one spatial dimension, and hence the capacity distribution would be easy to obtain. For a $\left(L_{t}, 1\right)$ MISO system, the capacity (23) becomes

$$
C_{\text {mimo }}\left(\mathbf{h}^{T}, \frac{\mathrm{SNR}}{L_{t}} \mathbf{I}_{L_{t}}\right)=\log \left(1+\frac{\mathrm{SNR}}{L_{t}} \sum_{i=1}^{L_{t}}\left|h_{i}\right|^{2}\right) .
$$

Define

$$
g_{L_{t}} \doteq \sum_{i=1}^{L_{t}}\left|h_{i}\right|^{2}
$$

the distribution of $C_{\text {mimo }}\left(\mathbf{h}^{T}, \frac{\mathrm{SNR}}{L_{t}} \mathbf{I}_{L_{t}}\right)$ is then completely characterized by the random variable $g_{L_{t}}$. If the attention is restricted to a Gaussian channel with $h_{i} \sim \mathcal{C N}(0,1)$, then $g_{L_{t}}$ follows a Chi-square distribution with $2 L_{t}$ degrees of freedom and it has a PDF

$$
f(g)=\frac{1}{\Gamma\left(L_{t}\right)} g^{L_{t}-1} e^{-g}, \quad g \geq 0,
$$

where $\Gamma\left(L_{t}\right)=\int_{0}^{\infty} t^{L_{t}-1} e^{-t} \mathrm{~d} t$ is the Gamma function. The CDF of $g_{L_{t}}, F_{G}(g) \doteq \operatorname{Pr}\left\{g_{L_{t}} \leq g\right\}=$ $\int_{0}^{\infty} f(t) \mathrm{d} t$, can also be calculated and a closed-form expression is given in [28]. The CDF of the MISO capacity then reduces to

$$
\operatorname{Pr}\left\{C_{\text {mimo }}\left(\mathbf{h}^{T}, \frac{\mathrm{SNR}}{L_{t}} \mathbf{I}_{L_{t}}\right) \leq R\right\}=\operatorname{Pr}\left\{g_{L_{t}} \leq \frac{\left(2^{R}-1\right) L_{t}}{\mathrm{SNR}}\right\}=F_{G}\left(\frac{\left(2^{R}-1\right) L_{t}}{\mathrm{SNR}}\right) .
$$

The equivalent IR channel capacity is given in (32), and the average rate with isotropic Gaussian input distribution for IR can now be re-written as

$$
\bar{R}_{\mathrm{ir}}=\sum_{n=1}^{N}\left(R^{(n)}-R^{(n+1)}\right)\left(1-F_{G}\left(\frac{\left(2^{R}-1\right) L_{t}}{\mathrm{SNR}}\right)\right) .
$$

\footnotetext{
${ }^{4}$ The case of SIMO $\left(L_{t}=1\right)$ can be similarly analyzed.
} 


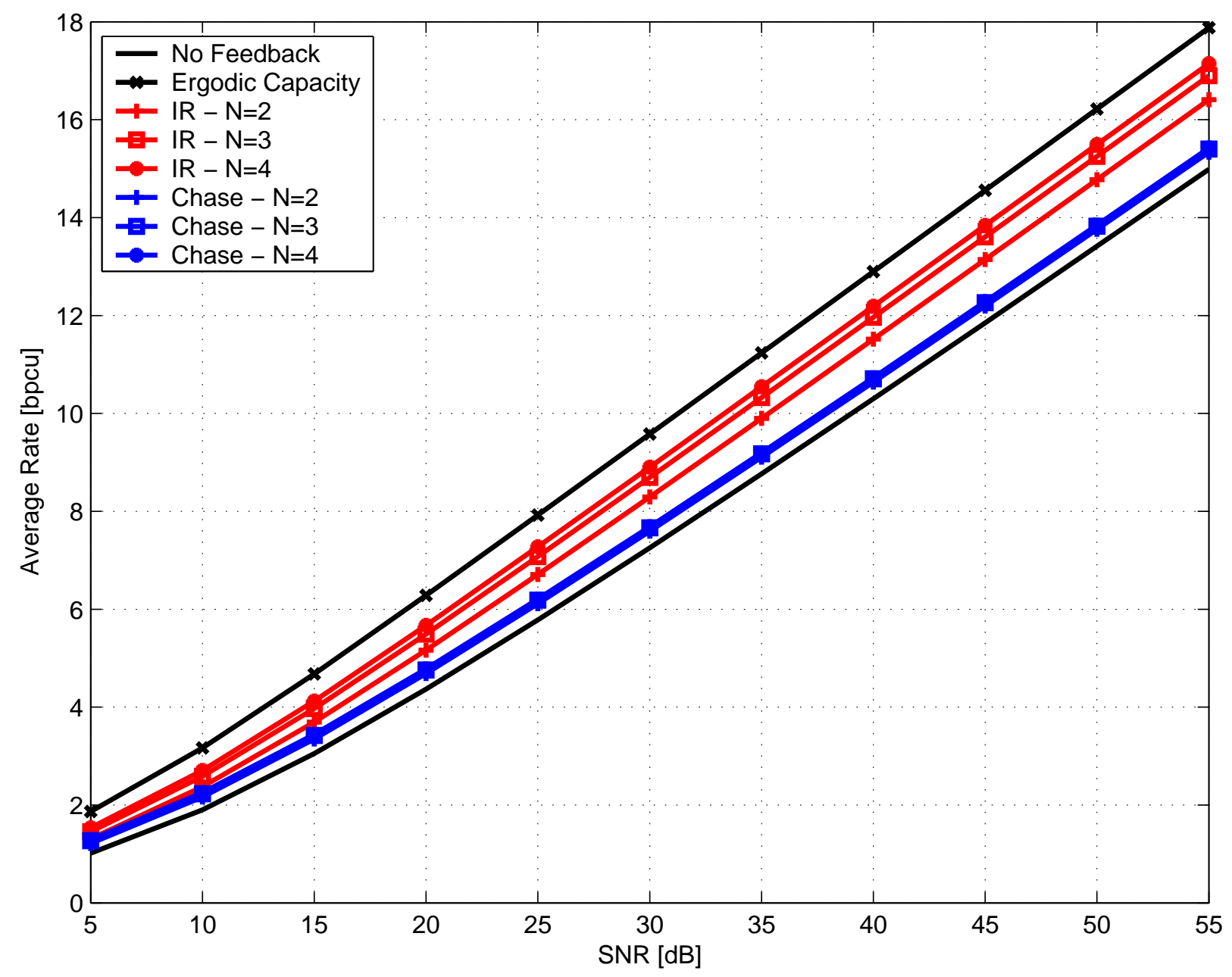

Figure 2: The optimal average rate performance of IR and CC in a $\left(L_{t}=2, L_{r}=1\right)$ MISO Gaussian channel with different $N$.

As for CC, the interesting observation is that the impact of each retransmission on the overall capacity is only to increase the receive $\mathrm{SNR}$ to $\mathrm{SNR}_{n}=n \mathrm{SNR}$. Thus,

$$
C_{\mathrm{cc}}^{(n)}\left(\mathbf{H}_{\mathrm{eq}, \mathrm{cc}}^{(n)}\right)=\frac{1}{n} \log \left(1+\frac{n \mathrm{SNR}}{L_{t}} g_{L_{t}}\right),
$$

and the average rate becomes

$$
\bar{R}_{\mathrm{cc}}=\sum_{n=1}^{N} \frac{R}{n}\left(F_{G}\left(\frac{\left(2^{R}-1\right) L_{t}}{(n-1) \mathrm{SNR}}\right)-F_{G}\left(\frac{\left(2^{R}-1\right) L_{t}}{n \mathrm{SNR}}\right)\right) .
$$

Unfortunately, such analytically tractable expressions are unavailable for the general MIMO channel, and we have resorted to numerical methods to solve the optimization problems. Fig. 2 and Fig. 3 give the numerical results of Problems (24) (29) for $\left(L_{t}=2, L_{r}=1\right)$ MISO and $\left(L_{t}=2, L_{r}=2\right)$ MIMO systems, respectively. The ergodic capacity and the optimal 


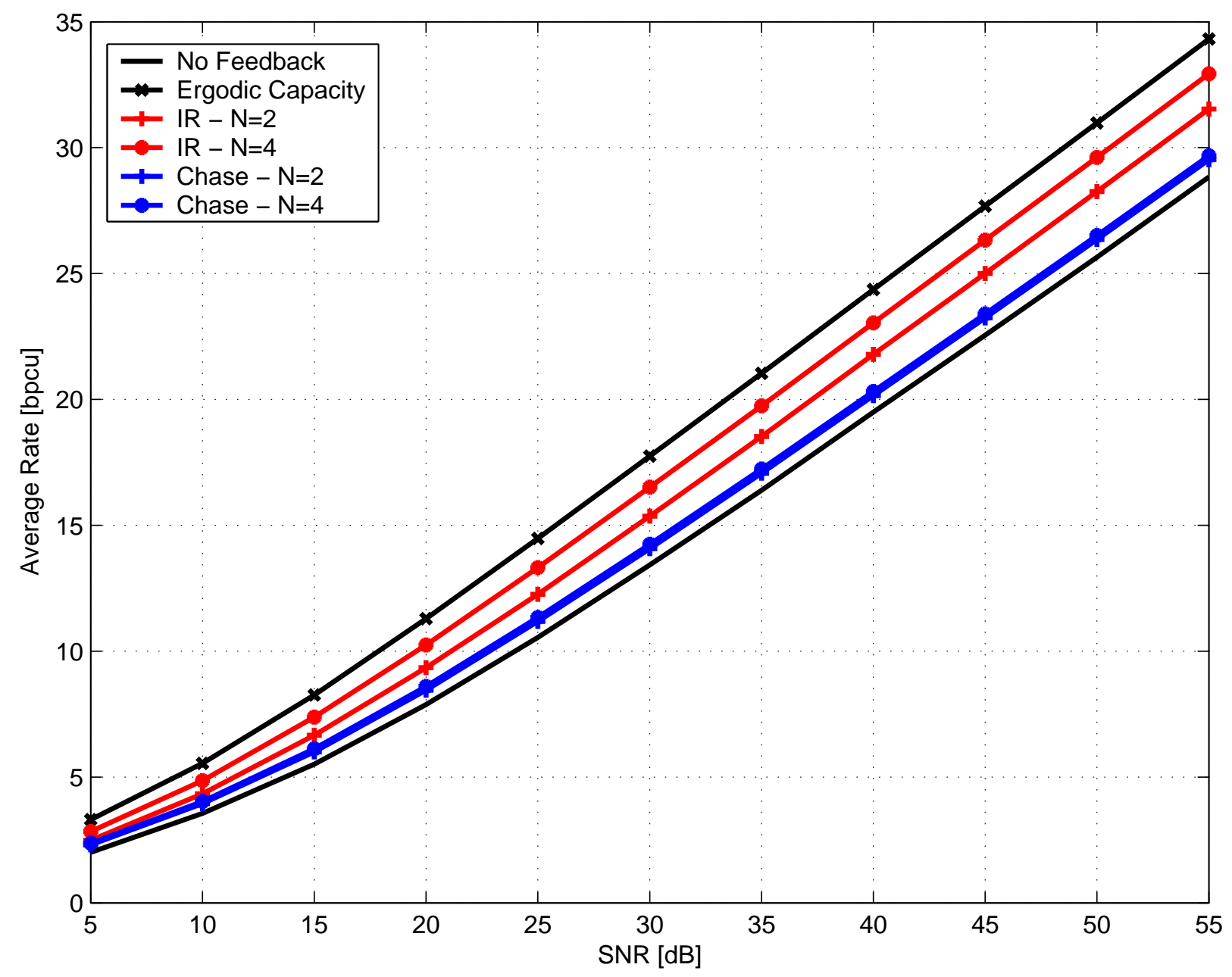

Figure 3: The optimal average rate performance of IR and CC in a $\left(L_{t}=2, L_{r}=2\right)$ MIMO Gaussian channel.

average rate of the no-feedback scheme are natural upper and lower bounds of the ARQ schemes [6], respectively, and they are plotted for reference. As one can see from the figures, IR performs better than the CC and no-feedback scheme and is close to the ergodic capacity. Moreover, the optimal average rate continues to increase as more ARQ rounds are allowed. In fact, the average rate of IR can be proved to asymptotically achieve the ergodic capacity, regardless of the fading distribution [6, Lemma 2]. On the other hand, the performance of $\mathrm{CC}$ is very limited, and increasing the maximum allowable ARQ rounds $N$ brings almost negligible gain to the optimal average rate. 


\section{Optimal LDC design: a mutual information analysis}

The previous section discusses the traditional IR and CC protocols and evaluates their performance. The IR protocol has excellent average rate thanks to two properties that allow full utilization of the ARQ retransmission. The first is the flexibility in rate assignment $\left\{R_{n}\right\}_{n=1}^{N}$, which can be accomplished by either the rate-compatible channel code via puncturing [4,29-34] or the recently developed rateless code [35-37]. On the other hand, the CC protocol has only one rate parameter $R$ to optimize, and each retransmission can reduce the rate by only an integer fraction. However, one should note that the advantage of IR rate assignment comes at the cost of complicated packetization: each transmission could have very different packet length, which is undesirable in practice. From this perspective, CC is preferred as each retransmission is a simple repetition of the previous packet. The second property is that each transmission in IR is capacity-achieving, provided that the channel coding and space-time transmission are carefully designed. For CC, however, it is clear from (26) that such capacity optimality is not valid in general, due to the repetition nature of this protocol. This also contributes to its poor performance.

This section introduces the MIMO-HARQ design based on STC, which combines the advantages of both IR and CC. The STC-based protocol has the IR capacity-achieving property for each retransmission, while still enjoys the repetition nature of $\mathrm{CC}$. Information-theoretic tools will be used in this section for the STC design of MIMO-HARQ. In Section 5, this design problem will be revisited from the decoding error probability perspective.

The idea can be best understood from the following simple example. Consider $L_{t}=2, L_{r}=1$ and $N=2$. For such configuration, the celebrated Alamouti code gives

$$
\mathbf{X}=\left[\begin{array}{cc}
s_{1} & -s_{2}^{*} \\
s_{2} & s_{1}^{*}
\end{array}\right]=\left[\mathbf{x}_{1}, \mathbf{x}_{2}\right]
$$

The traditional use of Alamouti code would transmit $\mathbf{X}$ in two channel uses. With ARQ feedback, however, one can separately transmit the first and second column. To be more specific, $\mathbf{x}_{1}$ is sent first. The receiver gets $y_{1}=h_{1} s_{1}+h_{2} s_{2}+z_{1}$ and jointly decodes $\left(s_{1}, s_{2}\right)$ in a maximum-likelihood (ML) manner. In case the first decoding attempt fails, a NACK will be sent back to the transmitter asking for the transmission of the second column $\mathbf{x}_{2}$. With both columns transmitted, the receiver can perform the usual Alamouti decoding to recover $s_{1}$ and $s_{2}$ in the second decoding attempt.

The idea behind this scheme is that if the channel matrix is "nice", it may be unnecessary to send a full-diversity ST codeword5. The first transmission, which only sends $\mathbf{x}_{1}$, is an aggressive attempt to exploit the channel. If the random channel is not good enough, we step back to the Alamouti scheme, with the help of ARQ feedback. It should be mentioned that HARQ schemes jointly considering STC and packet retransmission has been studied in the literature, e.g., $[15,17]$, but none of the designs are from an information-theoretic perspective

\footnotetext{
${ }^{5}$ This is further discussed at the end of Sec. 5.
} 
and hence do not give the best performance one can hope from STC-based HARQ. On the other hand, the information-theoretic studies of such schemes $[8,10]$ are mostly focused on the high SNR diversity-multiplexing-delay tradeoff and do not study the optimal STC design for a finite SNR.

\subsection{General framework}

The Linear Dispersion Code (LDC), proposed by Hassibi and Hochwald [38], is a general STC design that incorporates most of the known STBCs as special cases. This tool is used and a LDC framework is formulated to study the performance of STCs for the MIMO-HARQ design. Consider a $\left(L_{t}, L_{r}\right)$ MIMO system. For each LDC codeword $K$ modulated complex symbols are transmitted, and $T$ is used to denote the maximum number of time intervals of the LDC codeword for all ARQ rounds, i.e., $T=\sum_{n=1}^{N} T_{n}$. A LDC codeword can be expressed as a linear combination of the symbols 6

$$
\begin{aligned}
\mathbf{X} & =\sum_{k=1}^{K} \alpha_{k} \mathbf{A}_{k}+j \beta_{k} \mathbf{B}_{k} \\
& =\sum_{k=1}^{K} s_{k} \mathbf{C}_{k}+s_{k}^{*} \mathbf{D}_{k}
\end{aligned}
$$

where $s_{k}=\alpha_{k}+j \beta_{k}$ are the modulated symbols, and $\mathbf{A}_{k}, \mathbf{B}_{k}, \mathbf{C}_{k}, \mathbf{D}_{k} \in \mathcal{C}^{L_{t} \times T}$ are the LDC spreading matrices with

$$
\begin{aligned}
& \mathbf{A}_{k}=\mathbf{C}_{k}+\mathbf{D}_{k}, \\
& \mathbf{B}_{k}=\mathbf{C}_{k}-\mathbf{D}_{k} .
\end{aligned}
$$

For the HARQ transmission with a maximum $N$ rounds, the overall LDC codeword $\mathbf{X}$ shall be divided into $N$ sub-codewords

$$
\mathbf{X}=\left[\mathbf{X}_{1}, \mathbf{X}_{2}, \cdots, \mathbf{X}_{N}\right]
$$

where $\mathbf{X}_{n} \in \mathcal{C}^{L_{t} \times T_{n}}$ is for transmission at ARQ round $n$. The LDC spreading matrices can be similarly divided

$$
\begin{aligned}
& \mathbf{A}_{k}=\left[\mathbf{A}_{k, 1}, \mathbf{A}_{k, 2}, \cdots, \mathbf{A}_{k, N}\right] \\
& \mathbf{B}_{k}=\left[\mathbf{B}_{k, 1}, \mathbf{B}_{k, 2}, \cdots, \mathbf{B}_{k, N}\right],
\end{aligned}
$$

or

$$
\begin{aligned}
& \mathbf{C}_{k}=\left[\mathbf{C}_{k, 1}, \mathbf{C}_{k, 2}, \cdots, \mathbf{C}_{k, N}\right], \\
& \mathbf{C}_{k}=\left[\mathbf{D}_{k, 1}, \mathbf{D}_{k, 2}, \cdots, \mathbf{D}_{k, N}\right],
\end{aligned}
$$

\footnotetext{
${ }^{6}$ As our focus is to study the mutual information of the LDC structure with HARQ, the effect of channel coding length $L_{n}$ is irrelevant and is ignored in the discussion.
} 
where $\mathbf{A}_{k, n}, \mathbf{B}_{k, n}, \mathbf{C}_{k, n}, \mathbf{D}_{k, n} \in \mathcal{C}^{L_{t} \times T_{n}}$. Defining

$$
\begin{aligned}
T^{(n)} & =\sum_{i=1}^{n} T_{i}, \\
\mathbf{A}_{k}^{(n)} & =\left[\mathbf{A}_{k, 1}, \mathbf{A}_{k, 2}, \cdots, \mathbf{A}_{k, n}\right] \\
\mathbf{B}_{k}^{(n)} & =\left[\mathbf{B}_{k, 1}, \mathbf{B}_{k, 2}, \cdots, \mathbf{B}_{k, n}\right] \\
\mathbf{C}_{k}^{(n)} & =\left[\mathbf{C}_{k, 1}, \mathbf{C}_{k, 2}, \cdots, \mathbf{C}_{k, n}\right] \\
\mathbf{D}_{k}^{(n)} & =\left[\mathbf{D}_{k, 1}, \mathbf{D}_{k, 2}, \cdots, \mathbf{D}_{k, n}\right]
\end{aligned}
$$

the accumulated receive signal after the $n$-th transmission can be written as

$$
\mathbf{Y}^{(n)}=\sqrt{\frac{\mathrm{SNR}}{L_{t}}} \mathbf{H} \mathbf{X}^{(n)}+\mathbf{Z}^{(n)},
$$

with

$$
\begin{aligned}
\mathbf{X}^{(n)} & =\left[\mathbf{X}_{1}, \mathbf{X}_{2}, \cdots, \mathbf{X}_{n}\right] \\
& =\sum_{k=1}^{K} \alpha_{k} \mathbf{A}_{k}^{(n)}+j \beta_{k} \mathbf{B}_{k}^{(n)} \\
& =\sum_{k=1}^{K} s_{k} \mathbf{C}_{k}^{(n)}+s_{k}^{*} \mathbf{D}_{k}^{(n)}
\end{aligned}
$$

The capacity of the signal model (47) is denoted as $C_{\mathrm{ld}}^{(n)}(\mathbf{H})$, which is determined by the LD structure (48) or (49). Meanwhile, notice that the effective rate for ARQ round $n$ is

$$
R^{(n)}=\frac{R}{T^{(n)}}
$$

where $R$ is the channel coding rate, which is a design parameter. Now directly applying the general result (16), the average rate of LDC-based MIMO-HARQ can be expressed as

$$
\begin{aligned}
\bar{R}_{\mathrm{ld}} & =\sum_{n=1}^{N}\left(R^{(n)}-R^{(n+1)}\right) \operatorname{Pr}\left\{C_{\mathrm{ld}}^{(n)}(\mathbf{H}) \geq R^{(n)}\right\} \\
& =\sum_{n=1}^{N} \frac{R T_{n+1}}{T^{(n)} T^{(n+1)}} \operatorname{Pr}\left\{C_{\mathrm{ld}}^{(n)}(\mathbf{H}) \geq \frac{R}{T^{(n)}}\right\}
\end{aligned}
$$

where we use the convention $T^{(N+1)} \doteq \infty$ such that $R^{(N+1)}=0$, and $T_{N+1} / T^{(N+1)}=1$.

The general problem of average rate maximization for LDC-based MIMO-HARQ can be 
formally casted as

$$
\begin{array}{ll}
\underset{R,\left\{\mathbf{A}_{n}, \mathbf{B}_{n}, T_{n}\right\}_{n=1}^{N}}{\operatorname{maximize}} & \sum_{n=1}^{N} \frac{R T_{n+1}}{T^{(n)} T^{(n+1)}} \operatorname{Pr}\left\{C_{\mathrm{ld}}^{(n)}(\mathbf{H}) \geq \frac{R}{T^{(n)}}\right\} \\
\text { subject to } & R \geq 0 \\
& T_{n} \text { is a positive integer } \\
& \sum_{n=1}^{N} T_{n}=T \\
& \operatorname{Tr}\left(\sum_{k=1}^{K}\left(\mathbf{A}_{k, n} \mathbf{A}_{k, n}^{H}+\mathbf{B}_{k, n} \mathbf{B}_{k, n}^{H}\right)\right)=2 L_{t} T_{n}, \forall n=1, \cdots, N .
\end{array}
$$

Notice that $C_{\mathrm{ld}}^{(n)}(\mathbf{H})$ implicitly relies on $\left\{\mathbf{A}_{i}, \mathbf{B}_{i}, T_{i}\right\}_{i=1}^{n}$. The power constraint in Problem (52) will be discussed in more detail in Section 4.4.

\subsection{Design criterion}

Directly solving Problem (52), either analytically or numerically, is difficult, but there are some key properties that will help guide the optimal LDC design. The first observation is that the average rate is monotonic with the LDC capacity, and an achievable upper bound is the capacity of the MIMO channel. This leads to the following design criterion for the LDC design of MIMO-HARQ.

Criterion 1 (Capacity-based LDC design criterion) The LDC should satisfy

$$
C_{\mathrm{ld}}^{(n)}(\mathbf{H})=C_{\text {mimo }}\left(\mathbf{H}, \frac{\mathrm{SNR}}{L_{t}} \mathbf{I}_{L_{t}}\right)
$$

for any $A R Q$ round $n=1, \cdots, N$.

Notice that the intuition behind this criterion is that each LDC transmission should create an equivalent MIMO channel that is capacity lossless. Under the constraint of isotropic Gaussian distribution, this criterion gives the largest capacity for each transmission, which then minimizes the probability of decoding failure for any given $R$ and $\left\{T_{n}\right\}_{n=1}^{N}$.

With Criterion 1, the original Problem (52) is greatly simplified, as the dependence on $\left\{\mathbf{A}_{n}, \mathbf{B}_{n}\right\}_{n=1}^{N}$ disappears. The problem becomes how to find the optimal $R$ and $\left\{T_{n}\right\}_{n=1}^{N}$ that maximizes the average rate. In most of the remaining work a special case of $T_{n}=1$, $\forall n=1, \cdots, N$ is considered. There are two reasons to focus on this special situation.

1) Optimizing over all possible combinations of $\left\{T_{n}\right\}_{n=1}^{N}$ is complex even for moderate $N$. This requires solving a single-variable optimization problem for each possible combination of $\left\{T_{n}\right\}_{n=1}^{N}$ satisfying $\sum_{n=1}^{N} T_{n}=T$ and $T_{n}$ being a positive integer. The optimal solution may change with respect to the operating SNR or the channel distribution. In practice $\left\{T_{n}\right\}_{n=1}^{N}$ may be pre-determined with the choice of LDC, which leaves only one variable $R$ to optimize over. 
2) Choosing $T_{n}=1$ ensures that each ARQ round is of minimum delay, which is an important advantage. Meanwhile, this minimum delay may also result in the best throughput, as it is empirically observed as a good balance between $\frac{T_{n+1}}{T^{(n)} T^{(n+1)}}$ and $\operatorname{Pr}\left\{C_{\mathrm{ld}}^{(n)}(\mathbf{H}) \geq \frac{R}{T^{(n)}}\right\}$. Intuitively, large $T_{n}$ will result in a marginal gain in the decoding successful probability, but a significant decrease of the multiplicative coefficient.

To summarize, we give the following corollary.

Corollary 1 Assume isotropic Gaussian input distribution over all available transmit antennas. For a given set of $\left\{T_{n}\right\}_{n=1}^{N}$, the optimal average rate of a LDC-based MIMO-HARQ protocol is

$$
\bar{R}_{\mathrm{ld}}^{*}=\max _{R} \sum_{n=1}^{N} \frac{R T_{n+1}}{T^{(n)} T^{(n+1)}} \operatorname{Pr}\left\{C_{\text {mimo }}\left(\mathbf{H}, \frac{\mathrm{SNR}}{L_{t}} \mathbf{I}_{L_{t}}\right) \geq \frac{R}{T^{(n)}}\right\} .
$$

In the special case of $T_{n}=1, \forall n=1, \cdots, N$, the optimal average rate becomes

$$
\bar{R}_{\mathrm{ld}}^{*}=\max _{R} \sum_{n=1}^{N-1} \frac{R}{n(n+1)} \operatorname{Pr}\left\{C_{\text {mimo }}\left(\mathbf{H}, \frac{\mathrm{SNR}}{L_{t}} \mathbf{I}_{L_{t}}\right) \geq \frac{R}{n}\right\}+\frac{R}{N} \operatorname{Pr}\left\{C_{\text {mimo }}\left(\mathbf{H}, \frac{\mathrm{SNR}}{L_{t}} \mathbf{I}_{L_{t}}\right) \geq \frac{R}{N}\right\} .
$$

Criterion 1 and Corollary 1 are based on the mutual information of the equivalent MIMO channel. To more conveniently evaluate the optimality of any given LDC structure, it is helpful to develop a criterion that explicitly relies on the LDC spreading matrices $\left\{\mathbf{C}_{k}, \mathbf{D}_{k}\right\}_{k=1}^{K}$, which lead to Theorem 1 .

Theorem 1 Assume $L_{r} \geq L_{t}$ and $K=L_{t} T$. Define

$$
\begin{aligned}
\mathbf{U}^{(n)} & =\left[\operatorname{vec}\left(\mathbf{C}_{1}^{(n)}\right), \cdots, \operatorname{vec}\left(\mathbf{C}_{K}^{(n)}\right)\right] \\
\mathbf{V}^{(n)} & =\left[\operatorname{vec}\left(\mathbf{D}_{1}^{(n)}\right), \cdots, \operatorname{vec}\left(\mathbf{D}_{K}^{(n)}\right)\right]
\end{aligned}
$$

and

$$
\mathbf{F}^{(n)}=\left[\begin{array}{ll}
\mathbf{U}^{(n)} & \mathbf{V}^{(n)} \\
\operatorname{conj}\left(\mathbf{V}^{(n)}\right) & \operatorname{conj}\left(\mathbf{U}^{(n)}\right)
\end{array}\right] .
$$

The LDC-based HARQ protocol $\left\{\mathbf{C}_{k, n}, \mathbf{D}_{k, n}\right\}_{n=1 k=1}^{N}$ leads to the optimal average rate performance if and only if

$$
\mathbf{F}^{(n)} \mathbf{F}^{(n)^{H}}=\mathbf{I}_{2 L_{t} T^{(n)}},
$$

for all $n=1, \cdots, N$.

The proof follows almost directly from [39, Theorem 5.3.1]. The challenge is that Theorem 1 requires to consider $K>L_{t} T^{(n)}$ for each $n=1, \cdots, N-1$, while [39, Theorem 5.3.1] 
only holds for $K=L_{t} T$. However, a careful study of the proof of [39, Theorem 5.3.1] shows

that $\mathbf{F}^{(n)} \mathbf{F}^{(n)^{H}}=\mathbf{I}_{2 L_{t} T^{(n)}}$ would be necessary and sufficient for the LDC to be average rate optimal.

Theorem 1 considers the general LDCs that allow the conjugation operation. This is a key feature in some codes, e.g., Orthogonal STBC, but it has been argued [40] that there is not always a significant gain over complex LDCs, in which no conjugation operation is allowed. Hence, it is instructive to give the design criterion for complex LDCs, which follows directly from Theorem 1 .

Corollary 2 For a complex $L D C$

$$
\mathbf{X}^{(n)}=\sum_{k=1}^{K} s_{k} \mathbf{C}_{k}^{(n)}
$$

with $L_{r} \geq L_{t}$ and $K=L_{t} T$, it is average rate optimal if and only if

$$
\mathbf{U}^{(n)} \mathbf{U}^{(n)^{H}}=\mathbf{I}_{L_{t} T^{(n)}},
$$

for all $n=1, \cdots, N$.

\subsection{Evaluating existing LDCs}

Previous sections analyzed the optimal average rate performance of LDC-based MIMOHARQ and proposed the design criterion of the corresponding space-time structure. This section will attempt to use this general framework to evaluate existing LDC structures and their usage in the MIMO-HARQ setting. Although Theorem 1 and Corollary 2 provide straightforward means to evaluate given LDCs, we shall proceed to study the equivalent MIMO channel capacity and use Criterion 1 whenever it is feasible and simple. Directly evaluating the equivalent MIMO channel capacity and comparing to the physical channel capacity shed light on the (sub)optimality of the LDC. We will start with the simple MISO $L_{t}=2, L_{r}=1, N=2$ setting, in which analytical (sub)optimality can be rigorously shown. For the more complicated MIMO settings, numerical simulations are performed as a main tool to evaluate existing LDCs.

\subsubsection{MISO $L_{t}=2, L_{r}=1, N=2$}

This is the simplest MISO setting, in which the celebrated Alamouti code was invented [41]. The following LDC-based HARQ protocols are studied.

\section{Spatial Multiplexing with Repetition:}


This is the multiple-antenna version of the CC scheme. Spatial multiplexing vector $\mathbf{x}=$ $[\mathbf{x}(1), \mathbf{x}(2)]^{t}$ is repeated upon a NACK, and the overall codeword after $N=2$ transmissions is

$$
\left[\begin{array}{ll}
\mathbf{x}(1) & \mathbf{x}(1) \\
\mathbf{x}(2) & \mathbf{x}(2)
\end{array}\right] .
$$

Since ARQ round 1 is a spatial multiplexing, it is capacity-optimal:

$$
\begin{aligned}
C_{\mathrm{sm}}^{(1)}\left(\mathbf{h}^{t}\right) & =C_{\text {mimo }}\left(\left[\begin{array}{ll}
\mathbf{h}(1) & \mathbf{h}(2)
\end{array}\right], \frac{\mathrm{SNR}}{2} \mathbf{I}_{L_{t}}\right) \\
& =\log \left(1+\frac{\mathrm{SNR}}{2} g_{2}\right),
\end{aligned}
$$

where $g_{2}$ is defined in (33). The simple repetition in ARQ round 2, however, leads to a capacity loss:

$$
\begin{aligned}
C_{\mathrm{sm}}^{(2)}\left(\mathbf{h}^{t}\right) & =\frac{1}{2} C_{\text {mimo }}\left(\left[\begin{array}{ll}
\mathbf{h}(1) & \mathbf{h}(2) \\
\mathbf{h}(1) & \mathbf{h}(2)
\end{array}\right], \frac{\mathrm{SNR}}{2} \mathbf{I}_{L_{t}}\right) \\
& =\frac{1}{2} \log \left(1+\mathrm{SNR} g_{2}\right) \\
& =\frac{1}{2} C_{\text {mimo }}\left(\mathbf{h}^{t}, \mathrm{SNRI}_{L_{t}}\right) \\
& <C_{\text {mimo }}\left(\mathbf{h}^{t}, \frac{\mathrm{SNR}}{2} \mathbf{I}_{L_{t}}\right),
\end{aligned}
$$

for SNR $>0$, which proves the strict suboptimality of this protocol.

\section{Antenna Switching (AS):}

This scheme activates only one antenna at a time, and rotates the active antenna to achieve spatial diversity. The overall codeword is

$$
\left[\begin{array}{ll}
x & 0 \\
0 & x
\end{array}\right] .
$$

This is apparently a low-rate suboptimal scheme with

$$
\begin{aligned}
& C_{\text {as }}^{(1)}\left(\mathbf{h}^{t}\right)=\log \left(1+\mathrm{SNR} g_{1}\right)<C_{\text {mimo }}\left(\mathbf{h}^{t}, \frac{\mathrm{SNR}}{2} \mathbf{I}_{L_{t}}\right) \\
& C_{\mathrm{as}}^{(2)}\left(\mathbf{h}^{t}\right)=\frac{1}{2} \log \left(1+\mathrm{SNR} g_{2}\right)<C_{\text {mimo }}\left(\mathbf{h}^{t}, \frac{\mathrm{SNR}}{2} \mathbf{I}_{L_{t}}\right)
\end{aligned}
$$

and hence neither transmission is capacity-optimal.

\section{Alamouti Code:}


The overall codeword after $N=2$ transmissions is

$$
\left[\begin{array}{ll}
\mathbf{x}(1) & -\mathbf{x}(2)^{*} \\
\mathbf{x}(2) & \mathbf{x}(1)^{*}
\end{array}\right]
$$

The first transmission is a spatial multiplexing, which is optimal. It is also well known that the overall Alamouti structure also achieves the channel capacity [38]. Hence,

$$
C_{\text {Alamouti }}^{(1)}\left(\mathbf{h}^{t}\right)=C_{\text {Alamouti }}^{(2)}\left(\mathbf{h}^{t}\right)=C_{\text {mimo }}\left(\mathbf{h}^{t}, \frac{\mathrm{SNR}}{2} \mathbf{I}_{L_{t}}\right),
$$

and this protocol satisfies Criterion 1.

\section{Cyclic Delay Diversity (CDD):}

CDD was proposed in an OFDM setting [42] but the code itself presents an interesting space-time structure. It cyclically rotates the previously transmit column such that each symbol is sent from different antennas in different times slots. The overall codeword in the $\left(L_{t}=2, L_{r}=1\right)$ MISO is

$$
\left[\begin{array}{ll}
\mathbf{x}(1) & \mathbf{x}(2) \\
\mathbf{x}(2) & \mathbf{x}(1)
\end{array}\right]
$$

ARQ round 1 again is a spatial multiplexing, and hence is optimal. For ARQ round 2, the equivalent channel matrix is

$$
\mathbf{H}_{\text {eq, cdd }}=\left[\begin{array}{ll}
\mathbf{h}(1) & \mathbf{h}(2) \\
\mathbf{h}(2) & \mathbf{h}(1)
\end{array}\right]
$$

and

$$
\begin{aligned}
C_{\text {cdd }}^{(2)}\left(\mathbf{h}^{t}\right) & =\frac{1}{2} \log \operatorname{det}\left(\mathbf{I}_{2}+\frac{\mathrm{SNR}}{2} \mathbf{H}_{\text {eq, cdd }} \mathbf{H}_{\text {eq }, \text { cdd }}^{H}\right) \\
& =\frac{1}{2} \log \operatorname{det}\left[\begin{array}{cc}
1+\frac{\mathrm{SNR}}{2} g_{2} & \mathrm{SNR} \operatorname{Re}\left\{\mathbf{h}(1) \mathbf{h}(2)^{*}\right\} \\
\operatorname{SNR} \operatorname{Re}\left\{\mathbf{h}(1) \mathbf{h}(2)^{*}\right\} & 1+\frac{\mathrm{SNR}}{2} g_{2}
\end{array}\right] \\
& =\frac{1}{2} \log \left(1+\frac{\mathrm{SNR}}{2}|\mathbf{h}(1)+\mathbf{h}(2)|^{2}\right)+\frac{1}{2} \log \left(1+\frac{\mathrm{SNR}}{2}|\mathbf{h}(1)-\mathbf{h}(2)|^{2}\right) \\
& \leq \log \left(1+\frac{\mathrm{SNR}}{2}\left(|\mathbf{h}(1)|^{2}+|\mathbf{h}(2)|^{2}\right)\right) \\
& =C_{\text {mimo }}\left(\mathbf{h}^{t}, \frac{\mathrm{SNR}}{2} \mathbf{I}_{L_{t}}\right) .
\end{aligned}
$$

This suggests that the CDD-based protocol is suboptimal.

\section{Numerical Simulations:}

In addition to the previous analysis, numerical optimization is performed to obtain the average rates of several LDCs and the results are reported in Fig. 4. Optimal LDC, which 


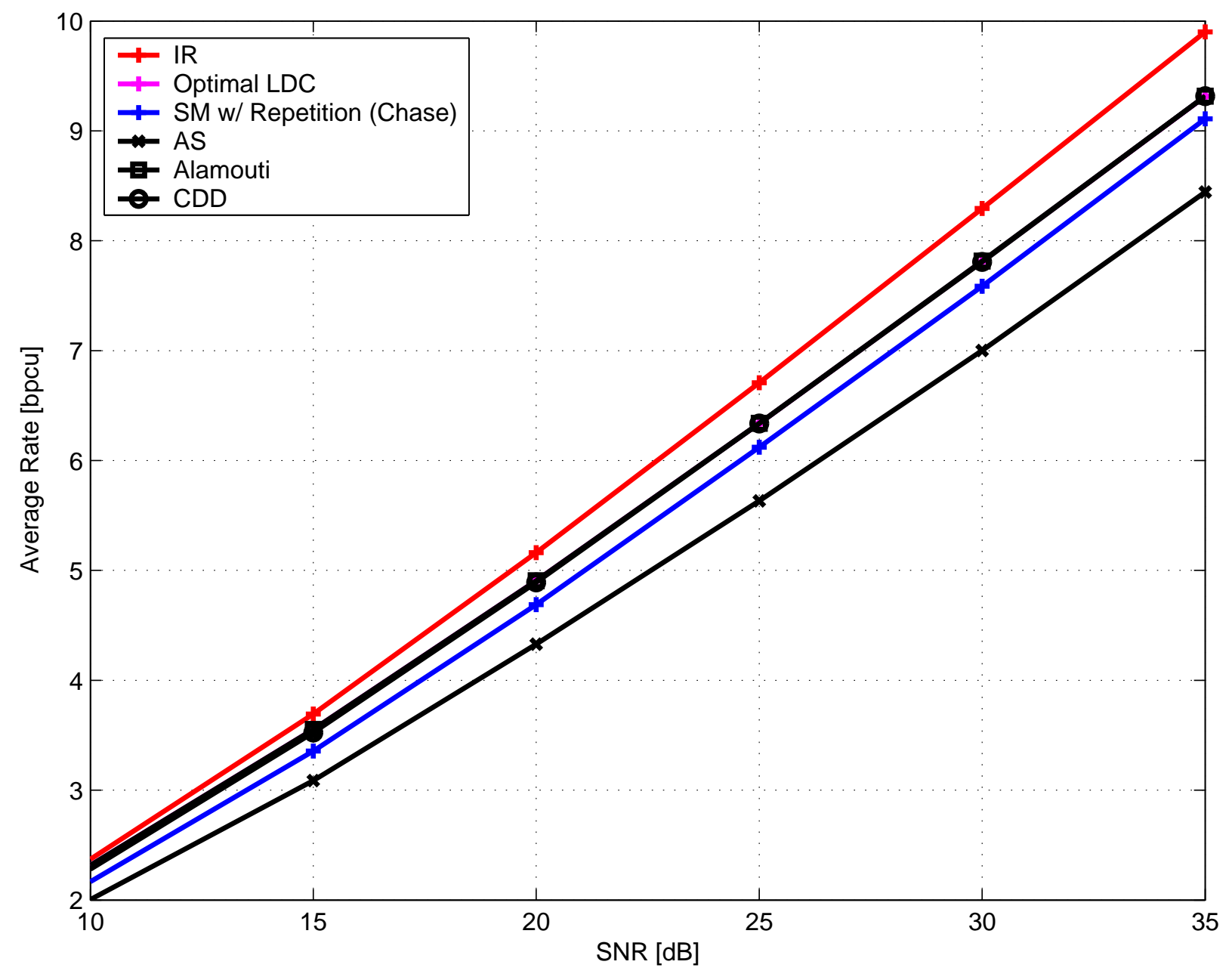

Figure 4: The optimal average rate performance of several existing LDCs in a $\left(L_{t}=2, L_{r}=\right.$ $1, N=2$ ) Gaussian MISO-HARQ channel.

satisfies Criterion 1, and IR are plotted as references. It is interesting to observe that although both AS and CDD are analytically proved to be sub-optimal, the average rate performance of CDD is very close to the optimum (almost negligible performance loss), while AS is extremely sub-optimal. This is no surprise as CDD utilizes the spatial degrees of freedom in a more efficient way than AS. SM-based repetition gives the performance of $\mathrm{CC}$ and is not far away from the optimal LDC-based protocol. Alamouti, as predicted in the analysis, is the optimal LDC.

\subsubsection{Full MIMO}

Analytically proving (sub)optimality becomes infeasible in most of the full MIMO settings, as the comparison of mutual information of a given LDC $\left\{\mathbf{A}_{n}, \mathbf{B}_{n}\right\}_{n=1}^{N}$ to the MIMO channel capacity is difficult to make even for moderate $N$. This section thus focuses on numerical 


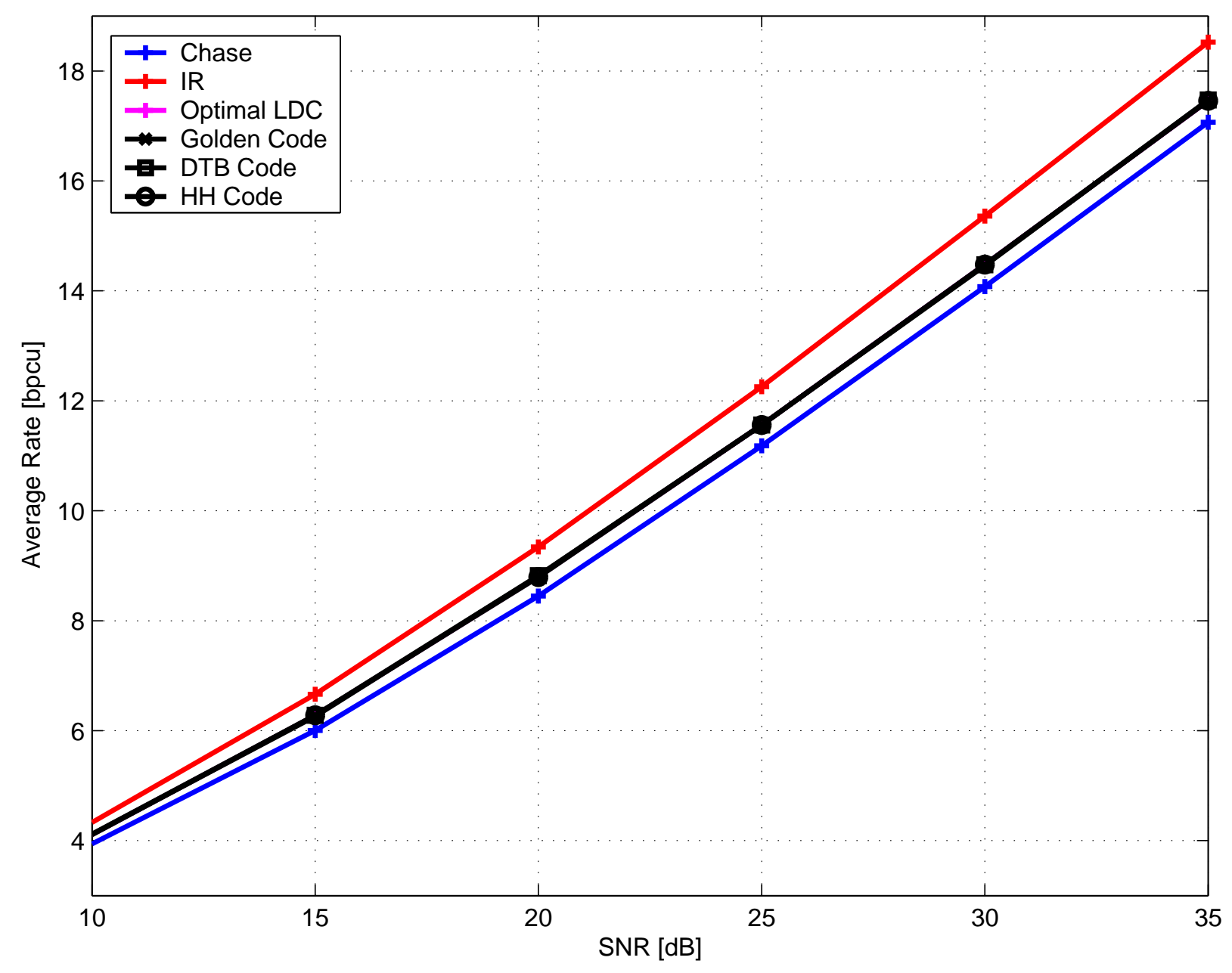

Figure 5: The optimal average rate performance of several existing LDCs in a $\left(L_{t}=2, L_{r}=\right.$ 2, $N=2$ ) Gaussian MIMO-ARQ channel.

simulations to get insight on existing LDCs.

Fig. 5 plots three well-known LDCs in a $\left(L_{t}=2, L_{r}=2\right)$ MIMO channel with $N=2$ : the Golden Code (Golden) [43], Damen, Tewfik and Belfiore's code (DTB) [44], and Hassibi and Hochwald's LD code (HH) [38, Equation (31)]. These three LDCs are known in the literature to be capacity optimal, and in fact it can be proven 7 that they satisfy Corollary 2 or Theorem 1, and is average rate optimal as well (the first column of the codeword is capacity lossless). Similar to the mutual information maximization approach of LDC design [38], typically there exist many LDCs that satisfy Criterion 1. In practice, one can search among these codes to further consider other criteria [40], such as the diversity and coding gain.

For a $\left(L_{t}=4, L_{r}=2\right)$ MIMO-HARQ system, Figs. [6and7 give the simulation results for $N=$ 2 and $N=4$, respectively. The LDCs under consideration are: Orthogonal STBC (OSTBC)

\footnotetext{
${ }^{7}$ This can be done by direcly verifying Corollary 2 or Theorem 1 using their corresponding LDC spreading matrices.
} 


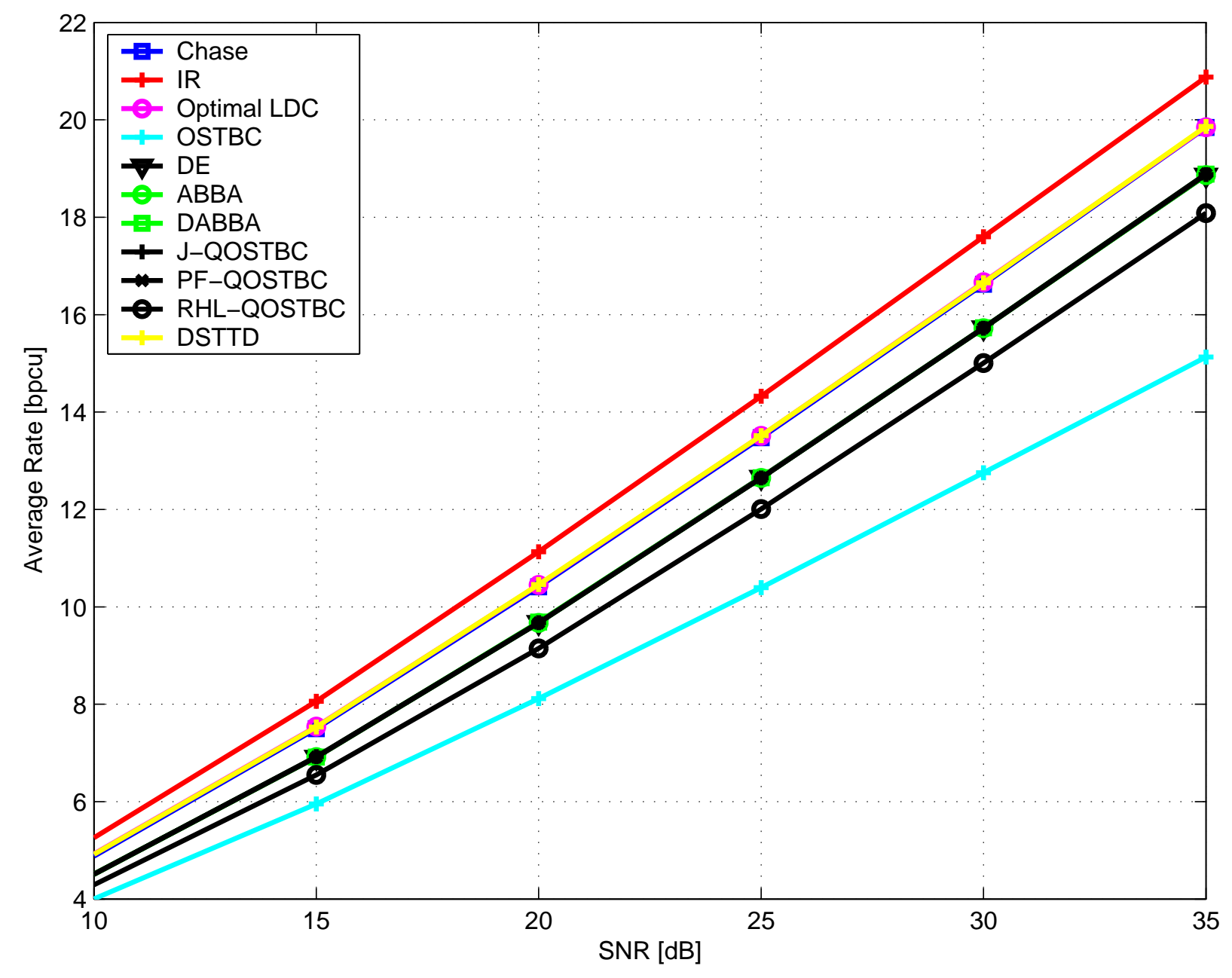

Figure 6: The optimal average rate performance of several existing LDCs in a $\left(L_{t}=4, L_{r}=\right.$ $2, N=2$ ) Gaussian MIMO-ARQ channel.

[45, Chapter 7.6], Diveristy Embedded STC (DE) [46], ABBA code [47], Double ABBA code (DABBA) [48], Jafarkhani's quasi-orthogonal STBC (J-QOSTBC) [49], Papadias and Foschini's QOSTBC (PF-QOSTBC) [50], Ran, Hou and Lee's QOSTBC (RHL-QOSTBC) [51], and Double Space-Time Transmit Diversity (DSTTD) [52]. CC, IR and optimal LDC are again plotted as reference curves.

Most existing LDCs for $L_{t}=4$ are obtained using $L_{t}=2$ LDCs as building blocks, which use 2 time slots. Hence, unlike other examples in this paper, the HARQ protocols for $N=2$ use $T_{1}=T_{2}=2$ and the results are reported in Fig. 6. The first observation is that even the optimal LDC has an average rate that is very close to CC. This suggests that with this specific configuration, one should not expect much average rate gain from LDC over simple repetition protocol. However, a well designed LDC-based protocol may have other advantages, such as diversity gain and simple decoding. The second conclusion is that among 


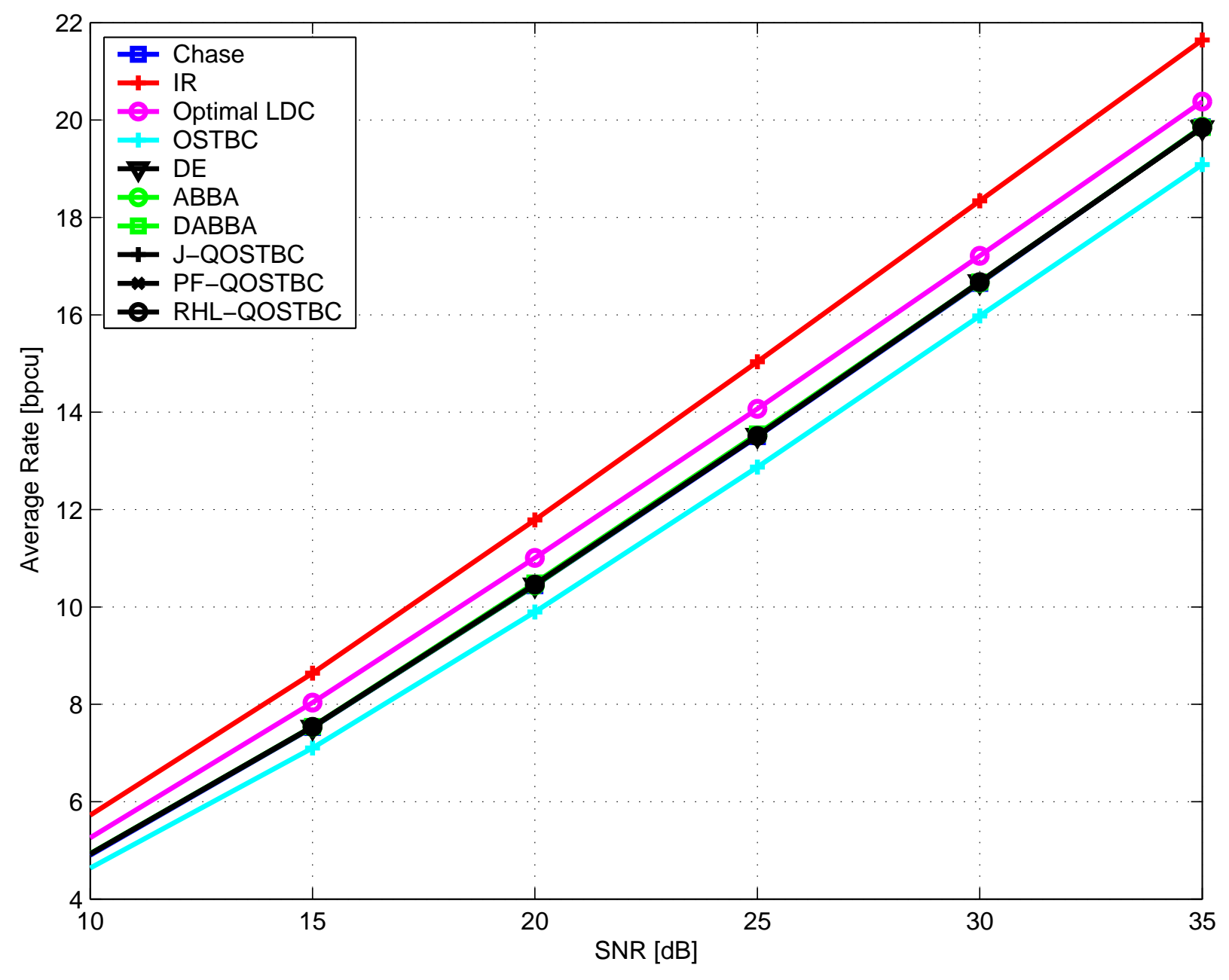

Figure 7: The optimal average rate performance of several existing LDCs in a $\left(L_{t}=4, L_{r}=\right.$ 2, $N=4$ ) Gaussian MIMO-ARQ channel. 
all the LDCs, only DSTTD performs close to the optimal LDC8. Among the other LDCs, five (ABBA, DABBA, DE, J-QOSTBC and PF-QOSTBC) have very similar optimal average rate, while RHL-QOSTBC is worse and OSTBC gives the worst performance. The extreme suboptimality of OSTBC is again due to its low-rate property (rate $3 / 4$ ).

The conclusions are different in the case of $N=4$, in which each ARQ round sends one column of the overall ST codeword matrix. From Fig. 7, there is a notable gain of optimal LDC over CC. Meanwhile, none of the above LDCs9 are optimal, which calls for the search of novel LDCs that approach the performance of optimal LDC.

\subsection{Remarks}

\section{Remark 1 Power Constraint}

We would like to constrain the long-term average transmit power in Problem (52). Two different types of power constraints can be considered.

\section{1) Each ARQ round has the same power.}

In this constraint, regardless of how many transmissions actually take place, the overall transmit power is constant due to the equal power allocation over ARQ rounds. The power constraint in the original problem (52) falls into this category:

$$
\operatorname{Tr}\left(\sum_{k=1}^{K}\left(\mathbf{A}_{k, n} \mathbf{A}_{k, n}^{H}+\mathbf{B}_{k, n} \mathbf{B}_{k, n}^{H}\right)\right)=2 L_{t} T_{n}, \forall n=1, \cdots, N .
$$

Note that with this constraint, each transmit antenna or each time slot can have different transmit power. Also the real and imaginary parts of the transmit constellation can have unequal power.

Similar to [38], more stringent power constraint can be posed to replace (72):

$$
\operatorname{Tr}\left(\mathbf{A}_{k, n} \mathbf{A}_{k, n}^{H}\right)=\operatorname{Tr}\left(\mathbf{B}_{k, n} \mathbf{B}_{k, n}^{H}\right)=\frac{L_{t} T_{n}}{K}
$$

or

$$
\mathbf{A}_{k, n} \mathbf{A}_{k, n}^{H}=\mathbf{B}_{k, n} \mathbf{B}_{k, n}^{H}=\frac{T_{n}}{K} \mathbf{I}_{L_{t}}
$$

where (73) ensures real and imaginary parts are transmitted with the same power, while (174) further forces energy to be spread equally in all spatial and temporal dimensions. These power constraints can be incorporated into Problem (52).

\footnotetext{
${ }^{8}$ It should be noted that DSTTD is in fact capacity suboptimal.

${ }^{9}$ DSTTD only uses two time slots and thus cannot be used with $N=4$.
} 


\section{2) Dynamic power allocation among ARQ rounds.}

Instead of always having a constant power for each ARQ round, one can further allow power allocation among the $N$ ARQ rounds. For example, giving more power to ARQ round 1 will increase the probability that the first transmission succeeds. However this comes at the price of decreasing the power of potential ARQ rounds 2 to $N$, if the overall average transmit power is kept constant. There is a serious challenge to maintain a constant average power. The reason is that except for the first transmission, ARQ rounds 2 to $N$ happen only with a non-one probability. This challenge has been addressed in the scalar channel case [6], and the solution is to average the power consumption with respect to the channel fading distribution.

Assuming that ARQ round $n$ has a total transmit power $\rho_{n}$, the actually consumed power $\rho$ is a random variable with $\mathrm{PMF}$

$$
\rho= \begin{cases}\frac{\sum_{i=1}^{n} \rho_{i} T_{i}}{\sum_{i=1}^{n} T_{i}}, & \text { if } \overline{\mathcal{A}_{1}}, \cdots, \overline{\mathcal{A}_{n-1}}, \mathcal{A}_{n} ; \forall n=1, \cdots, N-1 \\ \frac{\sum_{i=1}^{N} \rho_{i} T_{i}}{\sum_{i=1}^{N} T_{i}}, & \text { if } \overline{\mathcal{A}_{1}}, \cdots, \overline{\mathcal{A}_{N-1}} .\end{cases}
$$

Note that dynamic power allocation also affects the equivalent MIMO channel capacity after ARQ round $n: C_{\mathrm{ld}}^{(n)}(\mathbf{H})$ is a TDMA-type one where different portions of the code have different power [6].

To maintain an average total transmit power SNR, $\left(\rho_{1}, \cdots, \rho_{N}\right)$ should satisfy

$$
\sum_{n=1}^{N-1} \frac{\sum_{i=1}^{n} \rho_{i} T_{i}}{\sum_{i=1}^{n} T_{i}} \operatorname{Pr}\left\{\overline{\mathcal{A}_{1}}, \cdots, \overline{\mathcal{A}_{n-1}}, \mathcal{A}_{n}\right\}+\frac{\sum_{i=1}^{N} \rho_{i} T_{i}}{\sum_{i=1}^{N} T_{i}} \operatorname{Pr}\left\{\overline{\mathcal{A}_{1}}, \cdots, \overline{\mathcal{A}_{N-1}}\right\}=1
$$

Remark 2 Chase, $L D C$, and IR

Let us revisit the average rate for IR (24), CC (29), and LDC (52). It is straightforward to verify that if the rate assignment in IR is

$$
R^{(n)}=\frac{R}{T^{(n)}}, \quad T^{(n)} \text { is a positive integer, } \quad \forall n=1, \cdots, N,
$$

the average rate expression of IR and LDC will be the same, provided that Criterion 1 is satisfied. This demonstrates that the advantages of IR over LDC is the flexibility in rate assignment for each ARQ round, which comes at the cost of unequal packetization.

In reality, nevertheless, the freedom in rate assignment of IR is very limited due to practical limitations. Considerations such as packetization or slotted multi-user transmission usually ask for constant-length retransmissions, which leads to the same IR rate assignment as in LDC. With this constraint, the optimal average rates of IR and LDC are the same. In such cases, LDC has the potential advantage of receiver complexity over IR. Recall that IR requires code combining at the receiver - punctured codeword symbols need to be combined with the 
previous transmissions for another decoding. LDC-based protocol, on the other hand, only needs packet-level combining. Thus, the receiver complexity is shifted from channel decoding to front-end demodulation/detection. If the LDC is well designed, such detection complexity can be moderate, e.g., the Alamouti code. This can be an important advantage of LDC.

At the same time, LDC can also be viewed as an enhanced CC scheme. The reason is that in the LDC-based protocol each retransmission is essentially just a repetition of previously transmitted symbols - no new information is sent. However, the "repetition" in LDC is handled in a smart way such that the equivalent channel capacity is increased to approach the MIMO channel capacity, while the simple repetition CC scheme suffers from capacity loss. This can also be explained from the diversity perspective, which will be further developed in Sec. 5. The CC protocol naively repeats the same codeword upon each NACK, which cannot efficiently exploit the spatial diversity. A good LDC-based HARQ protocol spreads out the symbols onto different antennas upon each retransmission request to better exploit the spatial diversity. This becomes especially important in a quasi-static fading channel, as there is no time diversity to exploit.

\section{Remark 3 Switching Between Multiplexing and Diversity}

Compared to the STC-based HARQ scheme, there is a similar work [53] on switching between multiplexing and diversity, which is worth some comments. It is easy to see that the Alamouti example essentially performs a switching between multiplexing and diversity, just as [53] does. However, one can see that the ARQ-feedback-based approach is superior to the method in [53]. With ARQ feedback one actually sets the default choice to be full multiplexing. Hence, data communication takes place without even knowing whether one should use multiplexing or diversity. Switching to the diversity scheme only happens whenever necessary. This is possible because full multiplexing is embedded in any diversity schemes, which is not exploited in [53]. For the scheme in [53], the transmitter needs to wait for the feedback to inform which scheme to use before the data communication can take place. Another advantage of our approach is that ARQ feedback informs the decoding status to the transmitter, and the decision of switching to diversity scheme is made only when the receiver fails to decode the full multiplexing transmission. This is better than the selection criteria of [53], which use the minimum Euclidean distances and Demmel condition number of the MIMO channel to make the decision. Notice that the ultimate goal is to improve the decoding error performance, and hence decoding failure should be the best decision metric to determine switching. Our approach directly relies on the decoding error event, while [53] uses some indirect performance measures, which can only approximate the decoding error event. 


\section{Optimal LDC design: an error probability analysis}

Space-time codes design has followed two related but different paths. The original approach is from the viewpoint of detection theory. The focus is on studying the pariwise error probability (PWEP) [54-57], which has led to the well-known rank and determinant criteria that use diversity and coding gain to compare different STCs. A different approach is from the standpoint of information theory. It views STC as a modulation and study the optimal structure that preserves the channel mutual information $[38,58]$. There are also works that combine these two views $[39,53]$. The previous section investigated the optimal LDC design from an information-theoretic point of view. In this section, an error probability analysis of the optimal LDC-based HARQ protocol is performed, and the corresponding design criterion is presented.

\section{$5.1 \quad n$-th pairwise error probability}

Assume that the signal vector $\mathbf{s}=\left[s_{1}, \cdots, s_{K}\right]^{t}$ is chosen from a uniformly distributed set $\mathcal{S}$ with cardinality $M$ :

$$
\mathcal{S}=\left\{\mathbf{s}_{0}, \cdots, \mathbf{s}_{M-1}\right\}
$$

The probability of a decoding error after the $n$-th ARQ round can be written as

$$
\begin{aligned}
P_{e}^{(n)} & =\frac{1}{M} \sum_{j=0}^{M-1} \operatorname{Pr}\left\{\mathbf{s}^{(1)} \neq \mathbf{s}_{j}, \cdots, \mathbf{s}^{(n)} \neq \mathbf{s}_{j} \mid \mathbf{s}_{j} \text { was sent }\right\} \\
& =\frac{1}{M} \mathbb{E}\left[\sum_{j=0}^{M-1} \operatorname{Pr}\left\{\bigcup_{\substack{i_{1}=0 \\
i_{1} \neq j}}^{M-1} \cdots \bigcup_{\substack{i_{n}=0 \\
i_{n} \neq j}}^{M-1}\left\{Q_{i_{1}, j}^{(1)}<0, \cdots, Q_{i_{n}, j}^{(n)}<0\right\} \mid \mathbf{H}, \mathbf{s}_{j}\right\}\right] \\
& \leq \frac{1}{M} \sum_{j=0}^{M-1} \sum_{\substack{i_{1}=0 \\
i_{1} \neq j}}^{M-1} \cdots \sum_{\substack{i_{n}=0 \\
i_{n} \neq j}}^{M-1} \mathbb{E}\left[\operatorname{Pr}\left\{Q_{i_{1}, j}^{(1)}<0, \cdots, Q_{i_{n}, j}^{(n)}<0 \mid \mathbf{H}, \mathbf{s}_{j}\right\}\right]
\end{aligned}
$$

where (81) comes from the traditional union bound [59], $\mathbf{s}_{j}$ is the transmitted vector, $\mathbf{s}^{(n)}$ is the detected vector after ARQ round $n, Q_{i, j}^{(n)}$ is the pairwise decision metric in the $n$-th ARQ round, which is defined in the following. The optimum ML decoding rule for deciding between two possible codewords $\mathbf{X}^{(n)}\left(\mathbf{s}_{i}\right)$ and $\mathbf{X}^{(n)}\left(\mathbf{s}_{j}\right)$ for a given channel realization $\mathbf{H}$ and the receiver observation $\mathbf{Y}^{(n)}$ is

$$
\left\|\mathbf{Y}^{(n)}-\sqrt{\frac{\mathrm{SNR}}{L_{t}}} \mathbf{H X}^{(n)}\left(\mathbf{s}_{i}\right)\right\|_{F}^{2} \underset{\mathbf{s}_{j}}{\stackrel{\mathbf{s}_{i}}{<}}\left\|\mathbf{Y}^{(n)}-\sqrt{\frac{\mathrm{SNR}}{L_{t}}} \mathbf{H X}^{(n)}\left(\mathbf{s}_{j}\right)\right\|_{F}^{2} .
$$

Define the ML metric

$$
V_{i}^{(n)}=\left\|\mathbf{Y}^{(n)}-\sqrt{\frac{\mathrm{SNR}}{L_{t}}} \mathbf{H X}^{(n)}\left(\mathbf{s}_{i}\right)\right\|_{F}^{2},
$$




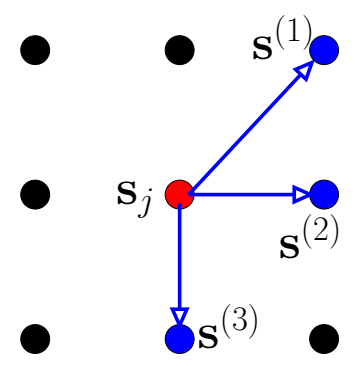

Figure 8: An example illustrating different error events in different ARQ rounds. $\mathbf{s}_{j}$ (red) is transmitted, and $\mathbf{s}^{(n)}$ is the ML detector output at ARQ round $n$, which are all erroneous (blue).

and $Q_{i, j}^{(n)}$ is defined as

$$
Q_{i, j}^{(n)} \doteq V_{i}^{(n)}-V_{j}^{(n)}
$$

For $n=1$, (81) reduces to the traditional union bound for STC:

$$
P_{e} \leq \frac{1}{M} \sum_{j=0}^{M-1} \sum_{\substack{i=0 \\ i \neq j}}^{M-1} \mathbb{E}\left[\operatorname{Pr}\left\{Q_{i, j}<0 \mid \mathbf{H}, \mathbf{s}_{j}\right\}\right]
$$

For Rayleigh fading,

$$
\operatorname{Pr}\left\{Q_{i, j}<0 \mid \mathbf{H}, \mathbf{s}_{j}\right\}=Q\left(\sqrt{\frac{d_{E}^{2}(i, j)}{2}}\right)
$$

where $d_{E}^{2}(i, j)$ is the squared Euclidean distance between two received codewords $\mathbf{Y}_{i}^{(n)}$ and $\mathbf{Y}_{j}^{(n)}$, with $\mathbf{Y}_{i}^{(n)} \doteq \sqrt{\frac{\mathrm{SNR}}{L_{t}}} \mathbf{H} \mathbf{X}^{(n)}\left(\mathbf{s}_{i}\right)$. Tools such as the Chernoff bound $[55,56]$ or the tighter bound in [57] can be applied. Meanwhile, assuming high SNR would further simplify the upper bound and it has led to the rank and determinant criteria.

The performance analysis of MIMO-HARQ, however, is much more involved. The first complication comes from (81). Unlike STC where only the PWEP is needed, analyzing MIMO-HARQ requires the $n$-th pairwise error probability ( $n$-PWEP):

$$
\operatorname{Pr}\left\{Q_{i_{1}, j}^{(1)}<0, \cdots, Q_{i_{n}, j}^{(n)}<0 \mid \mathbf{H}, \mathbf{s}_{j}\right\}
$$

in which the decision metrics $\left\{Q_{i_{l}, j}^{(l)}\right\}_{l=1}^{n}$ are correlated random variables. The complication is also intuitively understandable. The error event under consideration is when all the $n$ decoding attempts are erroneous. However, the decoding errors in different ARQ rounds could be very different (especially for short codes), depending on the channel realization and instantaneous noise. Fig. 8 is one such example. 
To analyze the $n$-PWEP, let us start with the statistics of $Q_{i, j}^{(n)}$. To simplify the derivation, define

$$
\mathbf{D}_{i, j}^{(n)} \doteq \sqrt{\frac{\mathrm{SNR}}{L_{t}}} \mathbf{H}\left(\mathbf{X}^{(n)}\left(\mathbf{s}_{i}\right)-\mathbf{X}^{(n)}\left(\mathbf{s}_{j}\right)\right)
$$

and

$$
\begin{aligned}
\mathbf{y}_{i}^{(n)} & \doteq \operatorname{vec}\left(\mathbf{Y}_{i}^{(n)}\right) \\
\mathbf{d}_{i, j}^{(n)} & \doteq \operatorname{vec}\left(\mathbf{D}_{i, j}^{(n)}\right)=\mathbf{y}_{i}^{(n)}-\mathbf{y}_{j}^{(n)} \\
\mathbf{z}^{(n)} & \doteq \operatorname{vec}\left(\mathbf{Z}^{(n)}\right) .
\end{aligned}
$$

Now conditioning on $\mathbf{H}$ and assuming $\mathbf{s}_{j}$ is transmitted, we have

$$
\begin{aligned}
Q_{i, j}^{(n)} & =\left\|\mathbf{D}_{i, j}^{(n)}+\mathbf{Z}^{(n)}\right\|_{F}^{2}-\left\|\mathbf{Z}^{(n)}\right\|_{F}^{2} \\
& =\left\|\mathbf{D}_{i, j}^{(n)}\right\|_{F}^{2}+2 \operatorname{Re}\left\{\operatorname{Tr}\left(\mathbf{D}_{i, j}^{(n)} \mathbf{Z}^{(n)}\right)\right\} \\
& =d_{E}^{(n)}(i, j)^{2}+W_{i, j}^{(n)}
\end{aligned}
$$

where $d_{E}^{(n)}(i, j)^{2}=\left\|\mathbf{D}_{i, j}^{(n)}\right\|_{F}^{2}$ is the squared Euclidean distance, and $W_{i, j}^{(n)} \doteq 2 \operatorname{Re}\left\{\operatorname{Tr}\left(\mathbf{D}_{i, j}^{(n)} \mathbf{Z}^{(n)^{H}}\right)\right\}$. It is easy to see that $W_{i, j}^{(n)}$ is a real Gaussian random variable

$$
\begin{aligned}
W_{i, j}^{(n)} & =\operatorname{Tr}\left(\mathbf{D}_{i, j}^{(n)} \mathbf{Z}^{(n)}{ }^{H}+\mathbf{D}_{i, j}^{(n)^{H}} \mathbf{Z}^{(n)}\right) \\
& =\mathbf{z}^{(n)^{H}} \mathbf{d}_{i, j}^{(n)}+\mathbf{d}_{i, j}^{(n)}{ }^{H} \mathbf{z}^{(n)}
\end{aligned}
$$

with

$$
\begin{aligned}
\mathbb{E}\left[W_{i, j}^{(n)}\right] & =0 \\
\mathbb{E}\left[W_{i, j}^{(n)^{2}}\right] & =\mathbb{E}\left[\mathbf{z}^{(n)^{H}} \mathbf{d}_{i, j}^{(n)}+\mathbf{d}_{i, j}^{(n)^{H}} \mathbf{z}^{(n)}\right] \\
& =\mathbb{E}\left[2 \mathbf{z}^{(n)^{H}} \mathbf{d}_{i, j}^{(n)} \mathbf{d}_{i, j}^{(n)}{ }^{H} \mathbf{z}^{(n)}\right] \\
& =2 d_{E}^{(n)}(i, j)^{2}
\end{aligned}
$$

which uses the fact that $\mathbf{Z}^{(n)}$ has complex circularly symmetric Gaussian entries with unit variance.

The $n$-PWEP now becomes

$\operatorname{Pr}\left\{Q_{i_{1}, j}^{(1)}<0, \cdots, Q_{i_{n}, j}^{(n)}<0 \mid \mathbf{H}, \mathbf{s}_{j}\right\}=\operatorname{Pr}\left\{W_{i_{1}, j}^{(1)}<-d_{E}^{(1)}\left(i_{1}, j\right)^{2}, \cdots, W_{i_{n}, j}^{(n)}<-d_{E}^{(n)}\left(i_{n}, j\right)^{2} \mid \mathbf{H}, \mathbf{s}_{j}\right\}$.

Notice that

$$
\mathbf{w}^{(n)} \doteq\left(W_{i_{1}, j}^{(1)}, \cdots, W_{i_{n}, j}^{(n)}\right)^{t}
$$


is an $n$-dimensional real Gaussian random vector. Thus, the only remaining problem is to obtain the statistics of $\mathbf{w}^{(n)}$. It is easy to get the mean

$$
\mathbb{E}\left[\mathbf{w}^{(n)}\right]=\mathbf{0} .
$$

As for the covariance matrix $\mathbb{E}\left[\mathbf{w}^{(n)^{2}}\right]$, the focus is on

$$
\mathbf{R}_{\mathbf{w}^{(n)}}(k, l) \doteq \mathbb{E}\left[W_{i_{k}, j}^{(k)} W_{i_{l}, j}^{(l)}\right], \forall l \geq k
$$

due to its symmetric property. The important observation is that

$$
\begin{aligned}
\mathbf{z}^{(l)} & =\operatorname{vec}\left(\mathbf{Z}^{(l)}\right) \\
& =\operatorname{vec}\left(\left[\mathbf{Z}^{(k)}, \mathbf{Z}_{l-k}\right]\right) \\
& =\left[\begin{array}{l}
\mathbf{z}^{(k)} \\
\mathbf{z}_{l-k}
\end{array}\right]
\end{aligned}
$$

and

$$
\begin{aligned}
\mathbf{d}_{i_{l}, j}^{(l)} & =\mathbf{y}_{i_{l}}^{(l)}-\mathbf{y}_{j}^{(l)} \\
& =\left[\begin{array}{l}
\mathbf{y}_{i_{l}}^{(k)}-\mathbf{y}_{j}^{(k)} \\
\mathbf{y}_{i_{l}, l-k}-\mathbf{y}_{j, l-k}
\end{array}\right] \\
& =\left[\begin{array}{l}
\mathbf{d}_{i_{l}, j}^{(k)} \\
\mathbf{d}_{i_{l}, j, l-k}
\end{array}\right] .
\end{aligned}
$$

Hence

$$
\begin{aligned}
\mathbf{R}_{\mathbf{w}^{(n)}}(k, l) & =\mathbb{E}\left[\left(\mathbf{z}^{(k)^{H}} \mathbf{d}_{i_{k}, j}^{(k)}+\mathbf{d}_{i_{k}, j}^{(k)}{ }^{H} \mathbf{z}^{(k)}\right)\left(\mathbf{z}^{(l){ }^{H}} \mathbf{d}_{i_{l}, j}^{(l)}+\mathbf{d}_{i_{l}, j}^{(l)}{ }^{H} \mathbf{z}^{(l)}\right)\right] \\
& =2 \operatorname{Re}\left\{\mathbb{E}\left[\mathbf{d}_{i_{k}, j}^{(k)}{ }^{H} \mathbf{z}^{(k)}\left(\mathbf{d}_{i_{l}, j}^{(l)}{ }^{H} \mathbf{z}^{(l)}\right)^{*}\right]\right\}
\end{aligned}
$$

which again uses the circularly symmetric property of the i.i.d complex Gaussian random matrix $\mathbf{Z}$. The following derivation is straightforward:

$$
\begin{aligned}
\mathbb{E}\left[\mathbf{d}_{i_{k}, j}^{(k)}{ }^{H} \mathbf{z}^{(k)}\left(\mathbf{d}_{i_{l}, j}^{(l)}{ }^{H} \mathbf{z}^{(l)}\right)^{*}\right] & =\mathbb{E}\left[\mathbf{d}_{i_{k}, j}^{(k)}{ }^{H} \mathbf{z}^{(k)}\left(\mathbf{d}_{i_{l}, j}^{(k)}{ }^{H} \mathbf{z}^{(k)}+\mathbf{d}_{i_{l}, j, l-k}{ }^{H} \mathbf{z}_{l-k}\right)^{*}\right] \\
& =\mathbb{E}\left[\mathbf{d}_{i_{k}, j}^{(k)}{ }^{H} \mathbf{z}^{(k)}\left(\mathbf{d}_{i_{l}, j}^{(k)} \mathbf{z}^{H}\right)^{(k)}\right] \\
& =\mathbf{d}_{i_{k}, j}^{(k)}{ }^{H} \mathbb{E}\left[\mathbf{z}^{(k)} \mathbf{z}^{(k)^{H}}\right] \mathbf{d}_{i_{l}, j}^{(k)} \\
& =\mathbf{d}_{i_{k}, j}^{(k)}{ }^{H} \mathbf{d}_{i_{l}, j}^{(k)} \\
& =\left\langle\mathbf{D}_{i_{k}, j}^{(k)}, \mathbf{D}_{i_{l, j}}^{(k)}\right\rangle_{F}
\end{aligned}
$$

where for matrices $\mathbf{A}$ and $\mathbf{B}$ of the same dimension,

$$
\langle\mathbf{A}, \mathbf{B}\rangle_{F} \doteq \operatorname{Tr}\left(\mathbf{A B}^{H}\right)
$$


is the Frobenius inner product [60], which induces the Frobenius norm. Equation (102) is because $\mathbf{z}^{(k)}$ and $\mathbf{z}_{l-k}$ are independent.

Now the covariance matrix $\mathbf{R}_{\mathbf{w}^{(n)}}$ is fully characterized as

$$
\begin{aligned}
\mathbf{R}_{\mathbf{w}^{(n)}}(k, l) & =2 \operatorname{Re}\left\{\left\langle\mathbf{D}_{i_{k}, j}^{(k)}, \mathbf{D}_{i_{l}, j}^{(k)}\right\rangle_{F}\right\}, \forall l>k \\
\mathbf{R}_{\mathbf{w}^{(n)}}(k, k) & =\left\|\mathbf{D}_{i_{k}, j}^{(k)}\right\|_{F}^{2}, \\
\mathbf{R}_{\mathbf{w}^{(n)}}(k, l) & =\mathbf{R}_{\mathbf{w}^{(n)}}(l, k), \forall l<k,
\end{aligned}
$$

and we conclude

$$
\mathbf{W}^{(n)} \sim \mathcal{N}\left(\mathbf{0}, \mathbf{R}_{\left.\mathbf{w}^{(n)}\right)} .\right.
$$

The $n$-dimensional joint Gaussian PDF of $\mathbf{w}^{(n)}$ is

$$
f_{\mathbf{w}^{(n)}}(\mathbf{w})=\frac{1}{(2 \pi)^{n / 2} \sqrt{\operatorname{det} \mathbf{R}_{\mathbf{w}^{(n)}}}} \exp \left(-\frac{1}{2} \mathbf{w}^{t} \mathbf{R}_{\mathbf{w}^{(n)}}{ }^{-1} \mathbf{w}\right) .
$$

In the existing literature of Gaussian tail distribution, much attention has been paid to either one- or two-dimensional Q functions [28]. It is clear that to analyze MIMO-HARQ with deadline $N$, one needs a general definition of the $n$-dimensional $Q$ function for a real Gaussian random vector $\mathbf{x} \sim \mathcal{N}\left(\mathbf{0}, \mathbf{R}_{\mathbf{x}}\right)$ (assuming zero mean without loss of generality)

$$
Q_{n}\left(\mathbf{x}, \mathbf{R}_{\mathbf{x}}\right) \doteq \frac{1}{(2 \pi)^{n / 2} \sqrt{\operatorname{det} \mathbf{R}_{\mathbf{x}}}} \int_{\mathbf{x}(1)}^{\infty} \cdots \int_{\mathbf{x}(n)}^{\infty} \exp \left(-\frac{1}{2} \mathbf{w}^{t} \mathbf{R}_{\mathbf{x}}{ }^{-1} \mathbf{w}\right) \mathrm{d} \mathbf{w} .
$$

Finally, the union bound on $P_{e}^{(n)}$ in (81) becomes

$$
P_{e}^{(n)} \leq \frac{1}{M} \sum_{j=0}^{M-1} \sum_{\substack{i_{1}=0 \\ i_{1} \neq j}}^{M-1} \cdots \sum_{\substack{i_{n}=0 \\ i_{n} \neq j}}^{M-1} \mathbb{E}_{\mathbf{H}}\left[Q_{n}\left(\mathbf{d}_{E}^{(n)^{2}}, \mathbf{R}_{\mathbf{w}^{(n)}}\right)\right],
$$

where

$$
\mathbf{d}_{E}^{(n)^{2}} \doteq\left(d_{E}^{(1)}\left(i_{1}, j\right)^{2}, \cdots, d_{E}^{(n)}\left(i_{n}, j\right)^{2}\right)^{t}
$$

\subsection{Design criterion}

The previous section studies the detection error probability after the $n$-th ARQ round. Using the traditional union bound shifts the focus to the $n$-PWEP. With the help of $n$-dimensional $\mathrm{Q}$ function and the distribution of the decision metrics, the $n$-PWEP is successfully calculated. However, the resulting union bound expression is complex and does not provide insight into the optimal LDC design. This section is devoted to developing the LDC-based HARQ design criterion from the detection error probability point of view. More specifically, 
the focus in on studying the diversity order of LDC-based HARQ10. The main result is stated in the following theorem.

Theorem 2 The optimum diversity order for any LDC-based HARQ protocol satisfies

$$
\operatorname{div}_{n}^{*}=L_{r} \min \left\{T^{(n)}, L_{t}\right\}
$$

for any $A R Q$ round $n, \forall n=1, \cdots, N$.

Proof: The following upper bound is straightforward:

$$
\operatorname{Pr}\left\{\overline{\mathcal{A}_{1}}, \cdots, \overline{\mathcal{A}_{n}}\right\} \leq \operatorname{Pr}\left\{\overline{\mathcal{A}_{n}}\right\} \text {. }
$$

Using this bound, the error probability (79) can be bounded as

$$
P_{e}^{(n)} \leq \frac{1}{M} \sum_{j=0}^{M-1} \operatorname{Pr}\left\{\mathbf{s}^{(n)} \neq \mathbf{s}_{j} \mid \mathbf{s}_{j} \text { was sent }\right\}
$$

The right hand side of (115) is in fact the detection error probability of a STC with codeword $\mathbf{X}=\mathbf{X}^{(n)} \in \mathcal{C}^{L_{t} \times T^{(n)}}$. The achievable diversity order for such STC is well-known to be $L_{r} \min \left\{T^{(n)}, L_{t}\right\}$. Hence

$$
\operatorname{div}_{n}^{*} \geq L_{r} \min \left\{T^{(n)}, L_{t}\right\} .
$$

On the other hand, the fundamental diversity order offered by the MIMO-ARQ channel has been established in $[8,9]$, which leads to

$$
\operatorname{div}_{n}^{*} \leq L_{r} \min \left\{T^{(n)}, L_{t}\right\}
$$

Combining (116) and (117) completes the proof.

Theorem 2 leads to the following diversity-driven LDC design criterion for MIMO-HARQ.

Criterion 2 (Error-probability-based LDC design criterion) The LDC should satisfy that after the $n$-th $A R Q$ round, $\forall n=1, \cdots, N$, the optimum detector has a diversity order $L_{r} \min \left\{T^{(n)}, L_{t}\right\}$.

\footnotetext{
${ }^{10}$ In this work, the diversity order is defined as

$$
\operatorname{div} \doteq-\lim _{\mathrm{SNR} \rightarrow \infty} \frac{\log P_{e}(\mathrm{SNR})}{\log \mathrm{SNR}}
$$

for a fixed-rate STC. This is the traditional definition of diversity, which is different from the one defined in [7] and many following DMT papers.
} 
This design criterion has some practical advantages over the capacity-based Criterion 1, as it is quite intuitive and easy to check. Meanwhile, it is also observed that those LDC-based HARQ protocols that satisfy Criterion 1 also tend to satisfy this diversity criterion.

Traditional STC design focuses on both the diversity and coding gain. A typical design would use full diversity as a constraint and then search for good coding gain. Our effort has been only on diversity due to the following two reasons. The first is that coding gain for MIMO-HARQ is not easy to define or compute, due to the complication in the $n$-PWEP. Theoretically, each ARQ round is equivalent to a STC $\mathbf{X}=\mathbf{X}^{(n)} \in \mathcal{C}^{L_{t} \times T^{(n)}}$, for which the coding gain can be computed. It is unclear how to optimize these $N$ correlated coding gains simultaneously. The second reason is that coding gain can be obtained from outer channel code as well. The diversity gain, however, has to be exploited by the proper LDC design. This is because the quasi-static channel is considered, and the only source of diversity is from the spatial domain.

\subsection{Numerical Examples and Discussions}

To gain more insight into the LDC-HARQ design based on the error probability analysis, we return to the Alamouti example (39) for $N=2$. Studying this simple example leads some interesting observations, which reveals the significance of ARQ feedback in different settings.

A software simulation system for $\left(L_{t}=2, L_{r}=1\right)$ MISO i.i.d. Gaussian quasi-static fading channel with maximum ARQ rounds $N=2$ is built. Alamouti-based protocol (39) is implemented, as well as the traditional repetition scheme (CC). Maximum-likelihood decoding is performed for both ARQ rounds, which gives the optimal decoding performance. We consider both uncoded and coded transmission with a Gray-coded QPSK constellation on each antenna. For the coded case, the standard 64 -state rate- $1 / 2$ binary convolutional code 11 with octal generators $(133,171)$ is implemented at the transmitter, which is followed by a standard row-in-column-out block interleaver. Each coded packet consists of 100 symbols. The receiver implements ML soft-output detectors with exact bit log-likelihood ratio (LLR) computation concatenated with a bit deinterleaver and a soft-input Viterbi algorithm convolutional decoder. Finally, it should be emphasized that as discussed in [6], the random channel generation in the simulation guarantees that the empirical channel distribution matches the true one.

Fig. 9 reports both the packet error rate and the average rate performance as a function of the average receive SNR. As has been analytically predicted in this work, the traditional ARQ approach which simply repeats the previous packet is significantly inferior to the Alamoutibased protocol in all settings. There is a clear diversity loss for the CC protocol, which also results in the degraded average rate performance. It should be remarked that the gains of Alamouti-based HARQ over $\mathrm{CC}$ are not only in packet error rate and average rate, but also

\footnotetext{
${ }^{11}$ The rate loss due to the termination bits is ignored.
} 
in the receiver soft-output ML detection complexity. Alamouti detector orthogonalizes the two transmitted symbols for independent bit LLR generation, while the CC protocol does not render such simple detector but an equivalent $2 \times 2$ MIMO system has to be formed to generate bit LLR, which is of higher complexity.

In general, the gains of HARQ protocols over direct transmission without ARQ feedback are two fold:

1) throughput;

2) decoding error probability.

The interesting observation from Fig. 9 is that these gains behave quite differently in different system configurations. The average rate gain for using ARQ feedback is remarkable for all considered protocols 12 , as can be seen in Fig. 9(b). However, the packet error rate gain of the two considered HARQ protocols over no-ARQ transmission is distinguishable for the uncoded case, but is almost negligible for the coded one, even if the channel coding is not capacity-approaching and the block length is short. This can be explained from a joint consideration of the mutual information analysis in Sec. 4 and the error probability analysis in Sec. 5. Let us take $N=2$ as an example. The decoding error probability of using ARQ is

$$
P_{\text {arq }}=\operatorname{Pr}\left\{\overline{\mathcal{A}_{1}}, \overline{\mathcal{A}_{2}}\right\},
$$

as compared to the no-ARQ case

$$
P_{\text {no-arq }}=\operatorname{Pr}\left\{\overline{\mathcal{A}_{2}}\right\}
$$

Hence, the gain of HARQ is

$$
\begin{aligned}
P_{\text {no-arq }}-P_{\text {arq }} & =\operatorname{Pr}\left\{\overline{\mathcal{A}_{2}}\right\}-\operatorname{Pr}\left\{\overline{\mathcal{A}_{1}}, \overline{\mathcal{A}_{2}}\right\} \\
& =\operatorname{Pr}\left\{\mathcal{A}_{1}, \overline{\mathcal{A}_{2}}\right\} \\
& \geq 0 .
\end{aligned}
$$

Notice that the event $\left\{\mathcal{A}_{1}, \overline{\mathcal{A}_{2}}\right\}$ means the receiver has a correct decoding in the first ARQ round, but it makes an incorrect decoding as it performs decoding based on both packets. This event is possible in the uncoded case, as there is a good probability that the noise realization in round 1 is small (hence the decoding is correct), but very large in the second round such that the decoding based on both transmissions fail. Recall that the channel transfer matrix keeps constant over ARQ rounds and hence the only source for this to happen is the noise realization. This explains the gain in the uncoded case. However, as the channel coding is implemented and the packet length is increased, the impact of finite noise realizations will eventually vanish. In fact, for the capacity-approaching coded transmission

\footnotetext{
${ }^{12}$ Some uncoded average rates seem to be better than coded ones in low SNR. This is because the coded packet is longer than the uncoded one, which counteracts the benefit from (short) channel coding.
} 


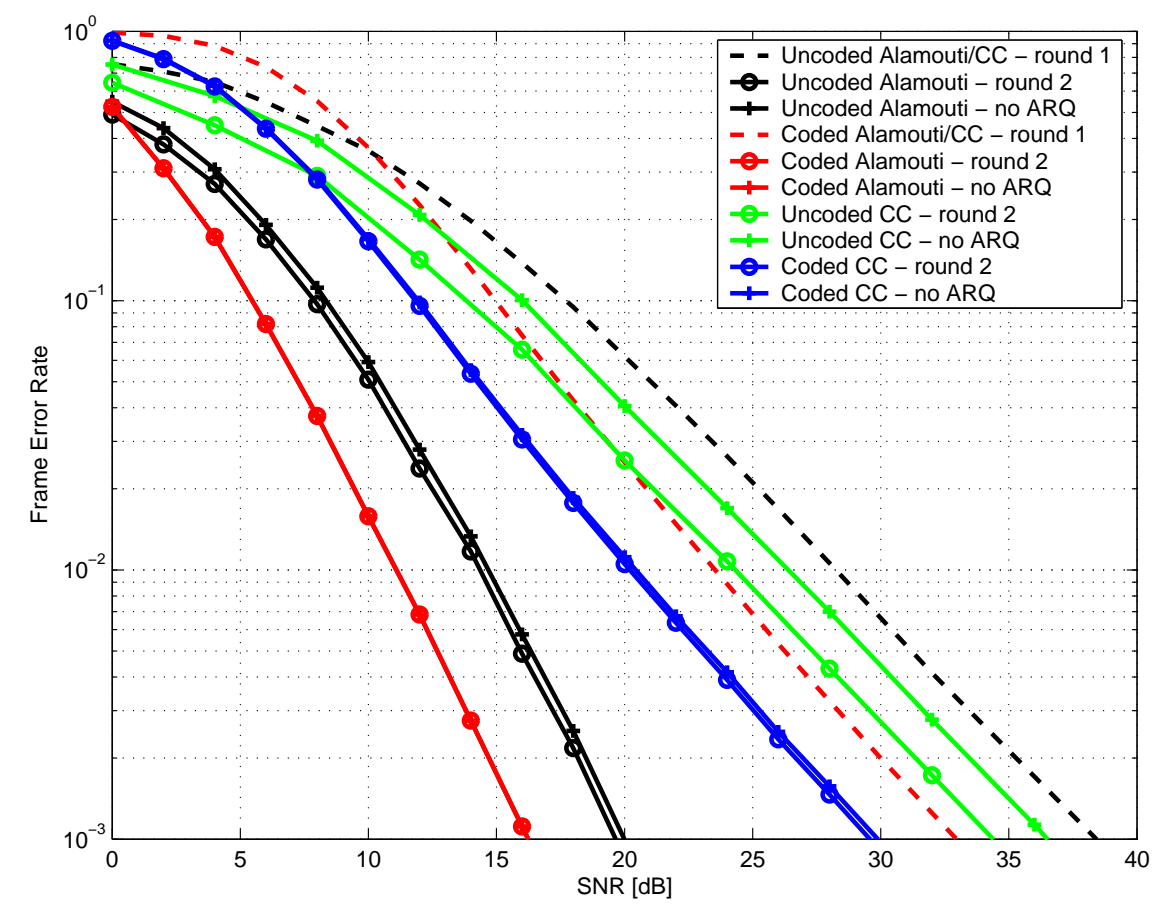

(a) PER

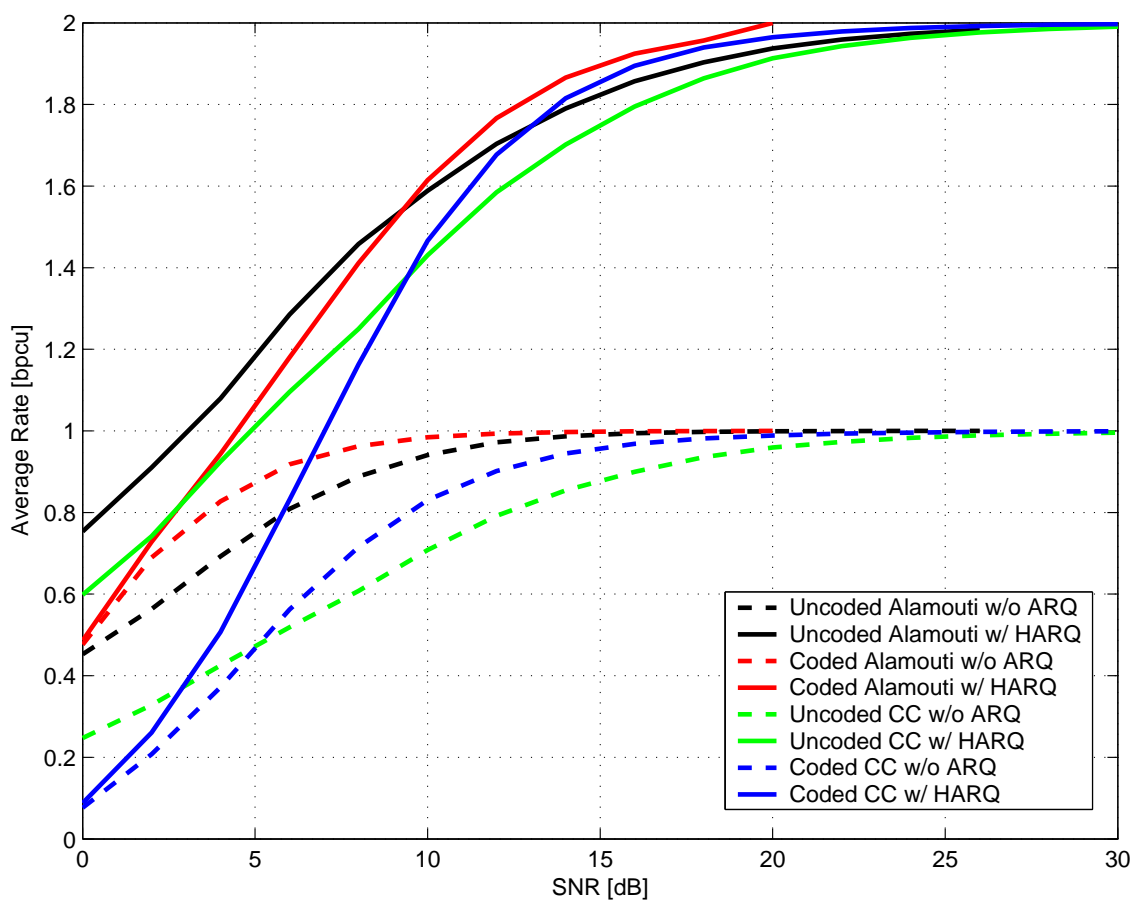

(b) Average Rate

Figure 9: Performance comparison of two HARQ protocols (Alamouti and CC, $N=2$ ) and no ARQ, with both uncoded and BICM coded transmissions in a $\left(L_{t}=2, L_{r}=1\right)$ MISO Gaussian channel. Fig. 9(a), Packet Error Rate (PER) versus SNR. Fig. 9(b); Average Rate (bits per channel use) versus SNR. 
as discussed in Sec. 3 and 4 , the noise realizations will be totally averaged out, which leads to Equation (8) and hence

$$
\operatorname{Pr}\left\{\mathcal{A}_{1}, \overline{\mathcal{A}_{2}}\right\}=0 \text {. }
$$

In summary, even with a relatively strong channel code with reasonably long block length, the two information-theoretic observations in Sec. 3 are generally valid, and the ARQ retransmission leads to only an average rate gain but very little error probability gain. On the other hand, very short coding length makes the ARQ retranmission even more beneficial, as the decoding error probability is improved prominently in addition to the average rate gain.

One might notice that there is only one degree of freedom for a $\left(L_{t}=2, L_{r}=1\right)$ MISO channel, but two independent symbols are sent in the first ARQ round. This observation reveals the essence of the HARQ gains. The concept of degrees of freedom is from the ergodic capacity, which averages over the channel distribution. For a certain subset of all possible channel realizations (together with appropriate noise realizations if the finite noise impacts the decoding), sending two independent symbols per channel use can result in a successful transmission. In this case, the spectral efficiency is doubled compared to the noARQ transmission, which is reflected in the average rate gain. If the channel realization is "bad", the second round transmission switches the entire protocol back to the normal STC transmission.

\section{Conclusions}

This paper studies the fundamental performance of Hybrid ARQ protocols in a multipleantenna channel. Unlike previous works that focus on the high SNR asymptotics, a general framework to optimize the average rate for any HARQ protocol with fixed SNR is presented. This general result is then applied to study two well-adopted HARQ protocols, incremental redundancy and packet combining (Chase). Space-time coded HARQ transmission is investigated under this framework, in which the capacity-based LDC design criterion is derived. Several existing LDCs are evaluated with this criterion, both analytically and numerically. A different design criterion based on the error probability analysis of LDC-HARQ is also presented. Numerical examples reveal different types of advantages of LDC-HARQ, and how they vary in different settings.

There are several interesting problems that have not been addressed in this work, which are the subjects of potential future work. For several MIMO and ARQ configurations, the existing LDCs are proved to be optimal in terms of average rate. However, there are some other settings where none of the known codes approach the optimal performance, e.g., the ( $\left.L_{t}=4, L_{r}=2, N=4\right)$ configuration in Fig. 7. Numerical design/search of optimal codes (e.g., similar to the approach in [38]) is an interesting research topic. Another possible direction is to incorporate the receiver decoding complexity into the LDC design criteria. Recall that the Alamouti-based protocol enjoys benefits of not only optimal average rate, excellent error performance, but also easy decoding in each ARQ rounds. Similarly, one can 
ask for the LDC to not only satisfy the design criteria presented in this work, but also has the "fast-decodable" property [61] for $\mathbf{X}^{(n)}$ in each ARQ round $n$. One might have noticed that for the LDC-based protocol to work, the channel has to remain constant over different ARQ rounds, which is the assumption of this paper. For time-varying channels, protocols exploiting both time and spatial diversity should be considered, and how to efficiently design the corresponding protocol could be another interesting problem.

\section{References}

[1] S. Lin, D. Costello, and M. Miller, "Automatic-repeat-request error-control schemes," IEEE Communications Magazine, vol. 22, no. 12, pp. 5-17, Dec. 1984.

[2] D. J. Costello, J. Hagenauer, H. Imai, and S. B. Wicker, "Applications of error-control coding," IEEE Trans. Info. Theory, vol. 44, no. 6, pp. 2531-2560, Oct. 1998.

[3] G. Caire and D. Tuninetti, "The throughput of Hybrid-ARQ protocols for the Gaussian collision channel," IEEE Trans. Info. Theory, vol. 47, no. 5, pp. 1971-1988, July 2001.

[4] S. Sesia, G. Caire, and G. Vivier, "Incremental redundancy Hybrid ARQ schemes based on low-density parity-check codes," IEEE Trans. Commun., vol. 52, no. 8, pp. 13111321, Aug. 2004.

[5] C. Shen, T. Liu, and M. Fitz, "Aggressive transmission with ARQ in quasi-static fading channels," in Proceedings of IEEE International Conference on Communications, May 2008, pp. 1092-1097.

[6] —, "On the average rate performance of Hybrid-ARQ in quasi-static fading channels," accepted to IEEE Trans. Commun., Apr. 2009. [Online]. Available: http://www.ee.ucla.edu/ congshen/ARQ_TCOM_revise2.pdf

[7] L. Zheng and D. Tse, "Diversity and multiplexing: a fundamental tradeoff in multipleantenna channels," IEEE Trans. Info. Theory, vol. 49, no. 5, pp. 1073-1096, May 2003.

[8] H. El Gamal, G. Caire, and M. O. Damen, "The MIMO ARQ Channel: diversitymultiplexing-delay tradeoff," IEEE Trans. Info. Theory, vol. 52, no. 8, pp. 3601-3621, Aug. 2006.

[9] T. Kim and M. Skoglund, "Diversity-Multiplexing tradeoff in MIMO channels with partial CSIT," IEEE Trans. Info. Theory, vol. 53, no. 8, pp. 2743-2759, Aug. 2007.

[10] A. Chuang, A. G. i Fabregas, L. K. Rasmussen, and I. B. Collings, "Optimal throughputdiversity-delay tradeoff in MIMO ARQ block-fading channels," IEEE Trans. Info. Theory, vol. 54, no. 9, pp. 3968-3986, Sept. 2008.

[11] E. N. Onggosanusi, A. G. Dabak, Y. Hui, and G. Jeong, "Hybrid ARQ transmission and combining for MIMO systems," in Proceedings of IEEE International Conference on Communications, vol. 5, May 2003, pp. 3205-3209. 
[12] M.-K. Oh, Y.-H. Kwon, and D.-J. Park, "Efficient Hybrid ARQ with space-time coding and low-complexity decoding," in Proceedings of IEEE International Conference on Acoustics, Speech, and Signal Processing, vol. 4, May 2004, pp. 589-592.

[13] E. Jang, J. Lee, H. Lou, and J. Cioffi, "Optimal combining schemes for MIMO systems with Hybrid ARQ," in Proceedings of IEEE International Symposium on Information Theory, June 2007, pp. 2286-2290.

[14] H. Zheng, A. Lozano, and M. Haleem, "Multiple ARQ processes for MIMO systems," in Proceedings of the 13th IEEE International Symposium on Personal, Indoor and Mobile Radio Communications, vol. 13, Sept. 2002, pp. 1023-1026.

[15] T. Koike, H. Murata, and S. Yoshida, "Hybrid ARQ scheme suitable for coded MIMO transmission," in Proceedings of IEEE International Conference on Communications, vol. 5, June 2004, pp. 2919-2923.

[16] S. Moon, H. Park, A. Goldsmith, and M. Oh, "Bit rearrangement for MIMO retransmissions," in Proceedings of IEEE Global Telecommunications Conference, Nov. 2007, pp. 3509-3513.

[17] E. de Carvalho and P. Popovski, "Strategies for ARQ in $2 \times 2$ MIMO systems," IEEE Commun. Letter, vol. 12, no. 6, pp. 441-443, June 2008.

[18] A. V. Nguyen and M. A. Ingram, "Hybrid ARQ protocols using space-time codes," in Proceedings of IEEE 54th Vehicular Technology Conference, vol. 4, 2001, pp. 2364-2368.

[19] K. Zheng, H. Long, L. Wang, and W. Wang, "Linear space-time precoder with Hybrid ARQ transmission," in Proceedings of IEEE Global Telecommunications Conference, Nov. 2007, pp. 3543-3547.

[20] S. Wicker, Error Control Systems for Digital Communication and Storage. Englewood Cliffs, NJ: Prentice-Hall, 1995.

[21] D. Chase, "Code combining-a maximum-likelihood decoding approach for combining an arbitrary number of noisy packets," IEEE Trans. Commun., vol. 33, no. 5, pp. 385-393, May 1985.

[22] M. Zorzi and R. R. Rao, "On the use of renewal theory in the analysis of ARQ protocols," IEEE Trans. Commun., vol. 44, no. 9, pp. 1077-1081, Sept. 1996.

[23] P. Wu and N. Jindal, "Performance of Hybrid-ARQ in block-fading channels: A fixed outage probability analysis," submitted to IEEE Trans. Commun., Nov. 2008. [Online]. Available: http://arxiv.org/abs/0811.4191

[24] Y. Liu, V. K. N. Lau, O. Y. Takeshita, and M. P. Fitz, "Optimal rate allocation for superposition coding in quasi-static fading channels," in Proceedings of IEEE International Symposium on Information Theory, Jun.-Jul. 2002, p. 111. 
[25] S. Shamai and A. Steiner, "A broadcast approach for a single-user slowly fading MIMO channel," IEEE Trans. Info. Theory, vol. 49, no. 10, pp. 2617-2635, Oct. 2003.

[26] M. Effros, A. Goldsmith, and Y. Liang, "Capacity definitions of general channels with receiver side information," in Proceedings of IEEE International Symposium on Information Theory, Jun. 2007, pp. 921-925.

[27] E. Telatar, "Capacity of multi-antenna Gaussian channels," AT\&T-Bell Labs, Tech. Rep., June 1995.

[28] M. K. Simon, Probability Distributions Involving Gaussian Random Variables: A Handbook for Engineers and Scientists. Kluwer Academic Publishers, 2002.

[29] J. Hagenauer, "Rate-compatible punctured convolutional codes (RCPC codes) and their applications," IEEE Trans. Commun., vol. 36, no. 4, pp. 389-400, Apr. 1988.

[30] J. Ha, J. Kim, and S. W. McLaughlin, "Rate-compatible puncturing of low-density parity-check codes," IEEE Trans. Info. Theory, vol. 50, no. 11, pp. 2824-2836, Nov. 2004.

[31] N. Varnica, E. Soljanin, and P. Whiting, "LDPC code ensembles for incremental redundancy Hybrid ARQ," in Proceedings of IEEE International Symposium on Information Theory, Sept. 2005, pp. 995-999.

[32] J. Ha, J. Kim, D. Klinc, and S. W. McLaughlin, "Rate-compatible punctured lowdensity parity-check codes with short block lengths," IEEE Trans. Info. Theory, vol. 52, no. 2, pp. 728-738, Feb. 2006.

[33] J. Kim, W. Hur, A. Ramamoorthy, and S. W. McLaughlin, "Design of rate-compatible irregular LDPC codes for incremental redundancy Hybrid ARQ systems," in Proceedings of IEEE International Symposium on Information Theory, Jul. 2006, pp. 1139-1143.

[34] C.-H. Hsu and A. Anastasopoulos, "Capacity achieving LDPC codes through puncturing," IEEE Trans. Info. Theory, vol. 54, no. 10, pp. 4698-4706, Oct. 2008.

[35] M. Luby, "LT codes," in Proceedings of the 43rd Annual IEEE Symposium on Foundations of Computer Science, 2002, pp. 271-282.

[36] A. Shokrollahi, "Raptor codes," IEEE Trans. Info. Theory, vol. 52, no. 6, pp. 2551-2567, Jun. 2006.

[37] E. Soljanin, N. Varnica, and P. Whiting, "Punctured vs rateless codes for Hybrid ARQ," in Proceedings of IEEE Information Theory Workshop, Punta del Este, Uruguay, Mar. 2006.

[38] B. Hassibi and B. M. Hochwald, "High-rate codes that are linear in space and time," IEEE Trans. Info. Theory, vol. 48, no. 7, pp. 1804-1824, Jul. 2002. 
[39] J. K. Zhang, J. Liu, and K. M. Wong, "Trace-orthogonal full diversity cyclotomic spacetime codes," in Space-Time Processing for MIMO Communications, A. Gershman and N. Sidiropoulos, Eds. John Wiley \& Sons, 2005, ch. 5, pp. 169-208.

[40] R. Heath and A. Paulraj, "Linear dispersion codes for MIMO systems based on frame theory," IEEE Trans. Signal Processing, vol. 50, no. 10, pp. 2429-2441, Oct. 2002.

[41] S. M. Alamouti, "A simple transmit diversity technique for wireless communications," IEEE J. Select. Areas Commun., vol. 16, no. 8, pp. 1451-1458, Oct. 1998.

[42] A. Dammann and S. Kaiser, "Performance of low complex antenna diversity techniques for mobile OFDM systems," in Proceedings of the 3rd International Workshop on MultiCarrier Spread-Spectrum \& Related Topics (MC-SS 2001), Oberpfaffenhofen, Germany, Sept. 2001, pp. 53-64.

[43] J.-C. Belfiore, G. Rekaya, and E. Viterbo, "The Golden Code: A 2x2 full-rate spacetime code with non-vanishing determinants," IEEE Trans. Info. Theory, vol. 51, no. 4, pp. 1432-1436, Apr. 2005.

[44] M. O. Damen, A. Tewfik, and J.-C. Belfiore, "A construction of a space-time code based on number theory," IEEE Trans. Info. Theory, vol. 48, no. 3, pp. 753-760, Mar. 2002.

[45] A. R. Calderbank and A. F. Naguib, "Introduction to space-time codes," in SpaceTime Wireless Systems: From Array Processing to MIMO Communications, H. Bolcskei, D. Gesbert, C. B. Papadias, and A.-J. van der Veen, Eds. Cambridge University Press, 2006, ch. 7, pp. 133-153.

[46] S. N. Diggavi, A. R. Calderbank, S. Dusad, and N. Al-Dhahir, "Diversity embedded spacectime codes," IEEE Trans. Signal Processing, vol. 54, no. 1, pp. 33-50, Jan. 2008.

[47] O. Tirkkonen, A. Boariu, and A. Hottinen, "Minimal non-orthogonality rate 1 spacetime block code for $3+$ tx antennas," in Proceedings of the Sixth IEEE International Symposium on Spread Spectrum Techniques and Applications (ISSSTA 2000), Sept. 2000, pp. 429-432.

[48] A. Hottinen and O. Tirkkonen, "Non-orthogonal space-time block code with symbol rate two," in Proceedings of Conference on Information Sciences and Systems (CISS), Baltimore, MD, USA, Mar. 2002.

[49] H. Jafarkhani, "A quasi-orthogonal space-time block code," IEEE Trans. Commun., vol. 49, no. 1, pp. 1-4, Jan. 2001.

[50] C. B. Papadias and G. J. Foschini, "Capacity-approaching space-time codes for systems employing four transmitter antennas," IEEE Trans. Info. Theory, vol. 49, no. 3, pp. 726-732, Mar. 2003.

[51] R. Ran, J. Hou, and M. Lee, "Triangular non-orthogonal space-time block code," in Proceedings of the 57th IEEE Vehicular Technology Conference, Apr. 2003, pp. 292295. 
[52] Texas Instruments, "Double-STTD scheme for HSDPA systems with four transmit antennas: Link level simulation results," TSG-R WG1 document TSGR1\#20(01)0458, May 2001.

[53] R. W. Heath and A. J. Paulraj, "Switching between diversity and multiplexing in MIMO systems," IEEE Trans. Commun., vol. 53, no. 6, pp. 962-968, June 2005.

[54] J.-C. Guey, M. P. Fitz, M. R. Bell, and W.-Y. Kuo, "Signal design for transmitter diversity wireless communication systems over Rayleigh fading channels," in 1996 IEEE Vehicular Technology Conference, Atlanta, GA, 1996, pp. 136-140.

[55] — - "Signal design for transmitter diversity wireless communication systems over Rayleigh fading channels," IEEE Trans. Commun., vol. 47, pp. 527-537, April 1999.

[56] V. Tarokh, N. Seshadri, and A. Calderbank, "Space-time codes for high data rate wireless communication: Performance criterion and code construction," IEEE Trans. Info. Theory, vol. 44, pp. 744-765, March 1998.

[57] S. Siwamogsatham, M. P. Fitz, and J. Grimm, "A new view of performance analysis of transmit diversity schemes in correlated Rayleigh fading," IEEE Trans. Info. Theory, vol. 48, no. 4, pp. 950-956, Apr. 2002.

[58] J. L. Massey, "Turbo codes, space-time codes, and what next?" in IEEE International Telecommunications Symposium (ITS2002), Sept. 2002.

[59] M. P. Fitz, Fundamentals of Communications Systems. McGraw-Hill, 2007.

[60] R. A. Horn and C. R. Johnson, Matrix Analysis. Cambridge University Press, 1985.

[61] E. Biglieri, Y. Hong, and E. Viterbo, "On fast-decodable space-time block codes," IEEE Trans. Info. Theory, vol. 55, no. 2, pp. 524-530, Feb. 2009. 